\title{
Chinese Stroke Association guidelines for clinical management of cerebrovascular disorders: executive summary and 2019 update of clinical management of ischaemic cerebrovascular diseases
}

To cite: Liu L, Chen W, Zhou H, et al. Chinese Stroke Association guidelines for clinical management of cerebrovascular disorders: executive summary and 2019 update of clinical management of ischaemic cerebrovascular diseases. Stroke \& Vascular Neurology 2020;5: e000378. doi:10.1136/ svn-2020-000378

- Additional material is published online only. To view, please visit the journal online (http://dx.doi.org/10.1136/svn2020-000378).

LL, WC and HZ contributed equally.

Received 17 March 2020 Revised 14 April 2020 Accepted 15 April 2020 Published Online First 18 June 2020

Check for updates

(c) Author(s) (or their employer(s)) 2020. Re-use permitted under CC BY-NC. No commercial re-use. See rights and permissions. Published by BMJ.

For numbered affiliations see end of article.

Correspondence to

Dr Yongjun Wang;

yongjunwang@ncrcnd.org.cn

\section{ABSTRACT}

Aim Stroke is the leading cause of disability and death in China. Ischaemic stroke accounts for about $60 \%-80 \%$ of all strokes. It is of considerable significance to carry out multidimensional management of ischaemic cerebrovascular diseases. This evidence-based guideline aims to provide the latest detailed and comprehensive recommendations on the diagnosis, treatment and secondary prevention of ischaemic cerebrovascular diseases.

Methods We had performed comprehensive searches of MEDLINE (via PubMed) (before 30 June 2019), and integrated the relevant information into charts and distributed to the writing group. Writing group members discussed and determined the recommendations through teleconference. We used the level of evidence grading algorithm of Chinese Stroke Association to grade each recommendation. The draft was reviewed by the Guideline Writing Committee of Chinese Stroke Association Stroke and finalised. This guideline is fully updated every 3 years.

Results This evidence-based guideline is based on the treatment, care and prevention of ischaemic cerebrovascular diseases, which emphasises on pathogenesis evaluation, intravenous thrombolysis, endovascular therapy, antiplatelet therapy, prevention and treatment of complications, and risk factor management. Conclusions This updated guideline presents a framework for the management of ischaemic cerebrovascular diseases. Timely first-aid measures, professional care in the acute stage, and proactive secondary prevention will be helpful to patients.

\section{INTRODUCTION}

Stroke is characterised by high disability, fatality and recurrence rate, and brings a huge burden to family and society. ${ }^{1-3}$ Although the incidence and prevalence of stroke have declined worldwide, there are 250 million new cases of a stroke every year in
China, and this number is still growing. The ischaemic cerebrovascular disease accounts for $60 \%-80 \%$ of stroke, including ischaemic stroke and transient ischaemic attack (TIA). There are many classification methodologies of ischaemic stroke. Using the Trial of Org 10172 in Acute Stroke Treatment (TOAST) criteria, ischaemic stroke can be divided into large artery atherosclerosis, cardioembolism, small artery occlusion, stroke of other determined aetiology and stroke of undetermined aetiology. ${ }^{56}$

It is of vital significance to identify and diagnose ischaemic stroke as early as possible. Several randomised controlled trials (RCTs) suggest that thrombolysis and endovascular therapy remarkably improve the prognosis of patients with acute ischaemic stroke. ${ }^{7-13}$ In addition, timely starting appropriate antithrombotic treatment in patient with minor stroke and high-risk TIA can reduce stroke recurrence rate without increasing the rate of haemorrhage and mortality (the Clopidogrel in High-Risk Patients With Acute NonDisabling Cerebrovascular Events (CHANCE), the Platelet-Oriented Inhibition in New TIA and Minor Ischemic Stroke (POINT) trials, etc) ${ }^{1415}$ In addition, the survival rate and prognosis of patients can be improved with timely intensive care, ${ }^{16}{ }^{17}$ rehabilitation, ${ }^{18}$ preventing and treating complications. ${ }^{19} 20$ Stroke is related to hypertension, diabetes, hyperlipidaemia, smoking, drinking and other correctable risk factors, improving living style consciously and monitoring chronic diseases regularly also help patients avoid recurrent stroke and long-term survival. ${ }^{21-25}$ 
The method of literature review, evaluation of level of evidence and grade of recommendation have all been published previously. This guideline is one of the seven guidelines organised by the Chinese Stroke Association on the recommendation of diagnosing and managing ischaemic cerebrovascular diseases. It contains nine sections: definitions, emergency assessment and diagnosis, reperfusion therapy, antiplatelet therapy, other treatment in the acute phase, general supportive care, complication management, early evaluation and diagnosis of aetiology and pathogenesis of ischaemic cerebrovascular disease, intervention on aetiology and pathogenesis, management of risk factors and long-term prevention. The full text of this guideline is available in the online supplementary material.

\section{SECTION 1: DEFINITIONS ASSOCIATED WITH ISCHAEMIC CEREBROVASCULAR DISEASES}

The definitions associated with ischaemic cerebrovascular diseases are shown in table 1 .

\section{SECTION 2: EMERGENCY ASSESSMENT AND DIAGNOSIS OF PATIENTS WITH ISCHAEMIC STROKE}

This section contains the recommendations for the management of acute ischaemic stroke (AIS), and indicates the main points of differential diagnosis.

The management flow chart of patients with AIS is shown in figure 1 .

\section{Recommendations}

1. It is recommended that patients suspected of having an ischaemic stroke should complete brain imaging within 30 min of arrival at the emergency department (class I, level of evidence B).

2. Emergency assessment of blood glucose, renal function, electrolytes, complete blood count (including platelet count), blood coagulation (including international normalised ratio (INR)), cardiac injury markers and a bedside 12-lead ECG is recommended, but should not delay the initiation of intravenous recombinant tissue plasminogen activator (IV rt-PA). For most patients, only the assessment of blood glucose must precede the initiation of IV rt-PA (class I, level of evidence B).

3. If feasible, patients with AIS within 6-24hours of last known normal who have large vessel occlusion (LVO) in the anterior circulation, obtaining CT perfusion (CTP) or diffusion-weighted imaging (DWI) with MRI perfusion is recommended to aid in patient selection for endovascular therapy. Patient selected for endovascular therapy should follow the same eligibility criteria of the two major RCTs (DWI or CTP Assessment With Clinical Mismatch in the Triage of Wake-Up and Late Presenting Strokes Undergoing Neurointervention With Trevo (DAWN) and Endovascular Therapy Fol-
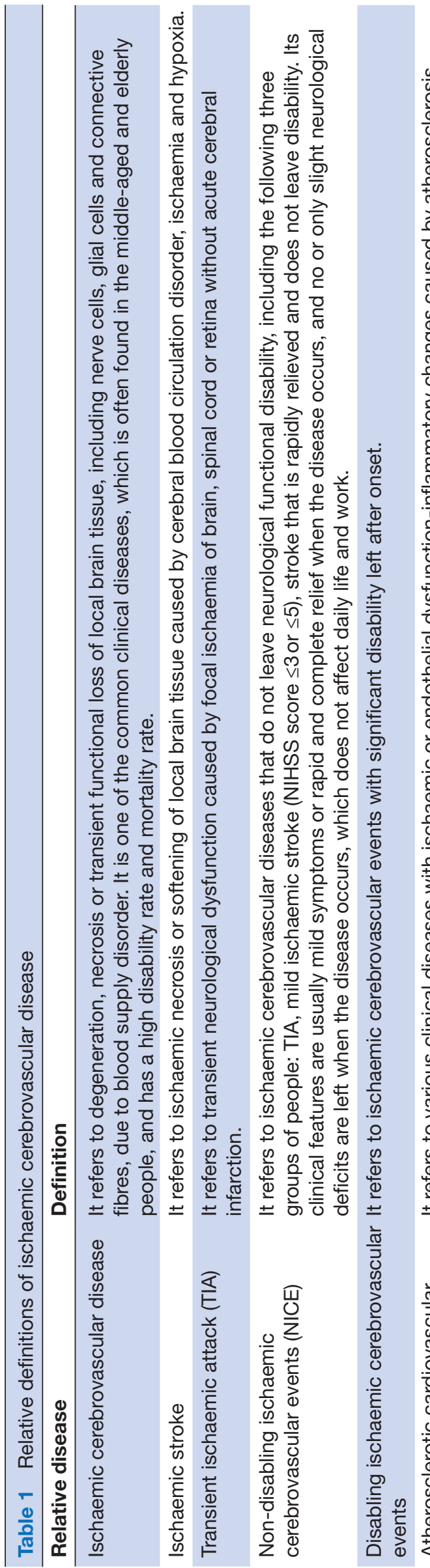


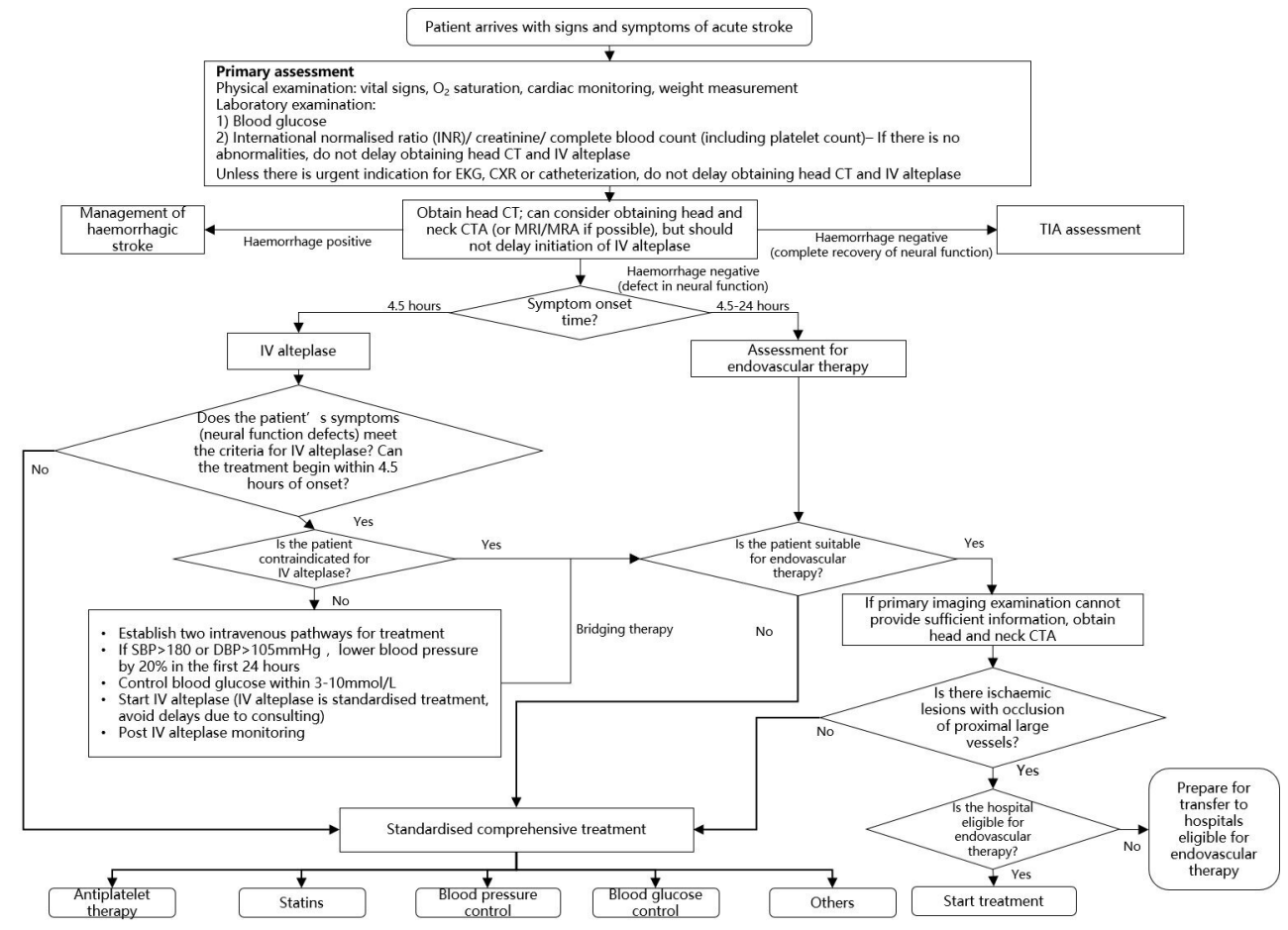

Figure 1 The management flow chart of patients with acute ischaemic stroke. CTA, CT angiography; CXR, chest X-ray; DBP, diastolic blood pressure; IV, intravenous; SBP, systolic blood pressure; TIA, transient ischaemic attack.

lowing Imaging Evaluation for Ischemic Stroke 3 (DEFUSE 3)) (class IIa, level of evidence B).

4. It is unclear whether using multimodal imaging criteria to select patients with ischaemic stroke who have unclear time of symptom onset for treatment with IV rt-PA is beneficial or not, therefore is not recommended outside a clinical trial (class III, level of evidence B).

5. If needed, multimodal imaging should be obtained as quickly as possible, to not delay administration of IV rt-PA (class IIb, level of evidence B).

6. It is unclear whether using perfusion imaging (CTP or perfusion weighted imaging) for selecting patients for endovascular treatment $<6$ hours is beneficial (class IIb, level of evidence B).

7. It is recommended for patients with AIS meeting the eligibility criteria of the two major RCTs (DAWN and DEFUSE 3) within 6-24hours of last known normal who have LVO in the anterior circulation to obtain CTP or DWI with MRI perfusion for subsequent endovascular therapy (class IIa, level of evidence B).

8. It is recommended that the Alberta Stroke Program Early CT Score (ASPECTS) based on head CT be considered when evaluating for endovascular treatment. However, the decision-making doctor must have received the training in the assessment of National Institutes of Health Stroke Scale (NIHSS) and ASPECTS scores and been verified for consistency (class IIa, level of evidence B).

9. For patients with acute presentation of neurological dysfunction, medical history record and physical examination must be performed rapidly. Medical history includes onset characteristics, predisposing factors, last known normal time, past medical history and current medication list. Physical examination includes vital signs and general physical examination. The use of NIHSS as a stroke severity rating scale is recommended (class I, level of evidence A).

\section{SECTION 3: REPERFUSION THERAPY FOR AIS}

For patients within the time window and without contraindications, thrombolytic therapy should be started as early as possible. When the patient has passed the time window for IV thrombolysis, or the symptoms are not relieved after thrombolysis, under the premise of meeting the criteria of DAWN or DEFUSE3, endovascular treatment can also be selected.

\section{Patients within 4.5 hours of onset of IV thrombolytic therapy}

The management process of IV thrombolysis in patients with ischaemic stroke within 4.5 hours of onset is shown in figure 2 .

\section{Recommendations}

1. Patients with elevated blood pressure and other aspects suitable for IV rt-PA therapy should be cautious in lowering blood pressure before thrombolysis. The recommended goal systolic blood pressure is $<180 \mathrm{~mm} \mathrm{Hg}$ and diastolic blood pressure is $<105 \mathrm{~mm}$ $\mathrm{Hg}$ (class I, level of evidence B).

2. It is reasonable to maintain blood pressure $(\leq 180 / 100 \mathrm{~mm} \mathrm{Hg})$ before intra-arterial therapy in patients who do not receive IV thrombolysis (class II, level of evidence B). 


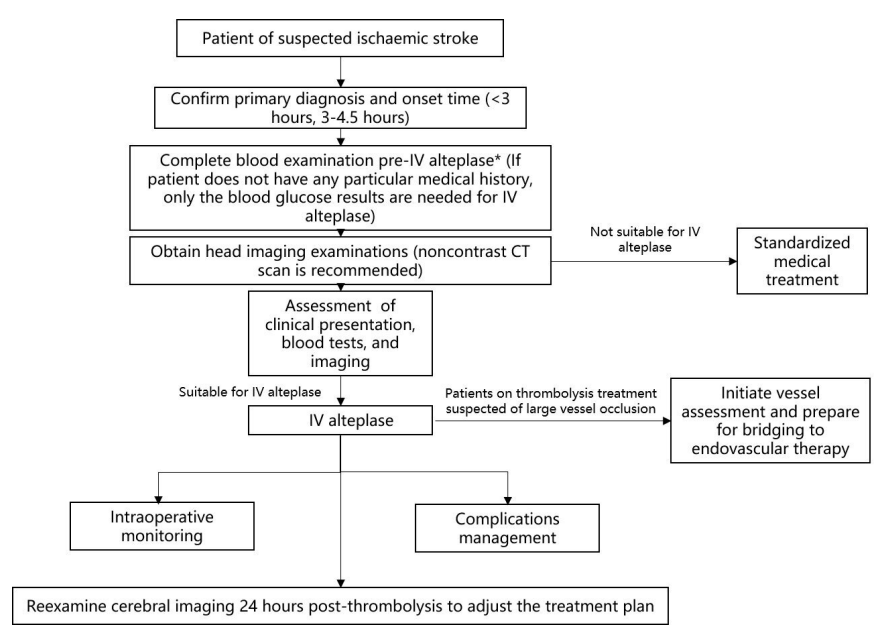

Figure 2 Intravenous recombinant tissue plasminogen activator (IV rt-PA) administration in patients with ischaemic stroke within 4.5 hours of onset.

3. Within 24 hours after IV rt-PA therapy, blood pressure should be $<180 / 105 \mathrm{~mm} \mathrm{Hg}$ (class I, level of evidence B).

4. Within 3 hours of onset, rt-PA IV thrombolytic therapy is recommended for patients aged over 18 years and who meet other criteria (class I, level of evidence A).

5. For patients who are suitable for IV thrombolysis within 3 hours of onset, IV rt-PA thrombolysis is recommended (drug dose $0.9 \mathrm{mg} / \mathrm{kg}$, maximum dose $90 \mathrm{mg}$, continuous infusion within $60 \mathrm{~min}$, of which $10 \%$ of the first dose is IV infusion within $1 \mathrm{~min}$ ) (class I, level of evidence A).

6. For patients with AIS with severe symptoms within 3 hours of onset, IV rt-PA thrombolysis is recommended. Although the risk of bleeding events increases, it still benefits (class I, level of evidence A).

7. For patients with mild symptoms but with disabling stroke symptoms within 3 hours of onset, IV rt-PA thrombolytic therapy is recommended. Current studies have shown that IV rt-PA thrombolytic therapy is beneficial for these patients (class I, level of evidence B).

8. IV rt-PA thrombolysis is still recommended for patients suitable for IV thrombolysis within 3-4.5 hours of onset (class I, level of evidence B).

9. The benefit of rt-PA thrombolytic therapy for patients with AIS aged over 80 years within 3-4.5 hours after onset is not clear (class IIb, level of evidence B).

10. Within 4.5 hours of AIS onset, low-dose IV rt-PA can be given to patients with potential high risk of haemorrhagic events. Usage: $\mathrm{rt}-\mathrm{PA} 0.6 \mathrm{mg} / \mathrm{kg}$ (maximum dose is $60 \mathrm{mg}$ ), of which $15 \%$ of the total amount was intravenously injected within the first $1 \mathrm{~min}$, and the remaining $85 \%$ was intravenously infused with infusion pump for 1 hour (class IIb, level of evidence B).

11. Considering the low incidence of platelet abnormalities and coagulation dysfunction in the general pop- ulation, IV thrombolysis should not be delayed while waiting for the results of platelet counts when there is no reason to suspect that the results of the tests are abnormal (class IIa, level of evidence B).

12. The safety and efficacy of IV rt-PA therapy in patients with AIS with potential haemorrhagic risk or coagulation disorders have not been determined (class III, level of evidence $\mathrm{C}$ ).

13. IV rt-PA is not suitable for patients who have used low molecular weight heparin (LMWH) within 24 hours, regardless of prophylactic or therapeutic dose (class III, level of evidence B).

14. Prethrombolytic MRI examination showed that IV thrombolysis was reasonable in patients with a number of (1-10) cerebral microbleeds (class IIa, level of evidence B).

15. Prethrombolytic MRI examination showed that IV thrombolysis was associated with an increased risk of symptomatic intracerebral haemorrhage in patients with a number of $(>10)$ cerebral microbleeds, and the clinical benefit is not clear. If there may be significant potential benefits, IV thrombolysis may be reasonable (class IIa, level of evidence B).

16. During IV thrombolysis, physicians should be fully prepared to respond to emergency adverse reactions, including haemorrhagic complications and vasogenic oedema that may cause airway obstruction (class I, level of evidence B).

17. Abciximab cannot be used concurrently with IV rt-PA (class III, level of evidence B).

18. Whether or not the endovascular treatment is bridged, the risk of starting antiplatelet therapy within 24 hours after IV thrombolysis remains unclear (class IIb, level of evidence B)

19. Urokinase is safe for those who are not suitable for rt-PA treatment within 6 hours of onset. However, the validity needs further confirmation by high-quality large sample size RCT (class IIb, level of evidence B).

20. It has not been confirmed that IV injection of tenecteplase (single dose of $0.4 \mathrm{mg} / \mathrm{kg}$ ) is superior or inferior to rt-PA. However, for patients with mild neurological dysfunction without occlusion of the intracranial artery, tenecteplase can be considered instead of rtPA (class IIb, level of evidence B).

21. In addition to clinical trials, ultrasound thrombolysis is not recommended as an adjunct therapy to IV thrombolysis. Desmoplatinolytic thrombolysis is not recommended under imaging guidance (class III, level of evidence B).

In some specific cases, high-level evidence is limited for the benefits or risks of thrombolysis. But most experts agree, as follows, for reference:

1. The time from onset to treatment has a major impact on prognosis, and IV rt-PA thrombolysis cannot be postponed to wait if symptoms are relieved (class IIa, level of evidence $\mathrm{C}$ ). 
2. For patients with mild non-disabling AIS, IV rt-PA therapy may be suitable within 3 hours of onset (class IIa, level of evidence C).

3. IV rt-PA may be beneficial for patients with AIS who had a history of digestive tract or urinary bleeding (class IIa, level of evidence $\mathrm{C}$ ).

4. AIS IV thrombolysis may be considered within 14 days after surgery, but should weigh the benefit of thrombolysis and the risk of haemorrhage of the surgical site (class IIb, level of evidence C).

5. In patients with AIS with recent major trauma history (within 14 days, without affecting the head), physician should carefully consider IV rt-PA treatment and should weigh the risk of the wound haemorrhage and the severity of stroke and subsequent disability (class IIb, level of evidence $\mathrm{C}$ ).

6. The safety and efficacy of IV rt-PA therapy in patients with AIS who have a history of vascular perforation within 7 days are not known (class IIb, level of evidence $\mathrm{C}$ ).

7. In patients with AIS with lumbar puncture within 7 days, the safety of IV rt-PA therapy is uncertain (class IIb, level of evidence C).

8. In patients with AIS with abnormal baseline glucose $(<50 \mathrm{mg} / \mathrm{dL} \quad(2.78 \mathrm{mmol} / \mathrm{L})$ or $>400 \mathrm{mg} / \mathrm{dL}$ $(22.2 \mathrm{mmol} / \mathrm{L}))$, followed by normalised blood glucose, the benefit of IV rt-PA is not determined (class IIb, level of evidence $\mathrm{C}$ ).

9. In patients with AIS with convulsions may benefit from IV rt-PA therapy if there is evidence that limb dysfunction is due to stroke rather than paralysis after seizures (class IIa, level of evidence $\mathrm{C}$ ).

10. In patients with AIS who have a known or suspected extracranial carotid artery dissection with an onset time $<4.5$ hours, IV rt-PA therapy should be chosen carefully (class IIa, level of evidence C).

11. In patients with AIS who have a known or suspected intracranial carotid artery dissection, the efficacy and safety of IV rt-PA therapy has not been established (class IIb, level of evidence C).

12. In patients with AIS with a small or moderate $(<10 \mathrm{~mm})$ unruptured intracranial aneurysm, IV rtPA therapy may be considered (class IIa, level of evidence $\mathrm{C}$ ).

13. In patients with AIS with large unruptured or unstable intracranial aneurysms, the risk and effectiveness of IV rt-PA thrombolysis is uncertain (class IIb, level of evidence $\mathrm{C}$ ).

14. In patients with AIS with unruptured or untreated intracranial vascular malformations, the safety and risk of IV rt-PA therapy is not known (class IIb, level of evidence $\mathrm{C}$ ).

15. Patients with AIS with neuroectodermal tumours may benefit from IV rt-PA thrombolysis (class IIa, level of evidence $\mathrm{C}$ ).

16. Patients with AIS with acute myocardial infarction may be considered for IV thrombolysis with the appropriate rt-PA dose of AIS, followed by percutane- ous coronary intervention or stent for acute coronary syndrome (class IIa, level of evidence C).

17. In patients with AIS with recent myocardial infarction (>3 months), rt-PA thrombolysis may be beneficial if there is also a non-ST elevation myocardial infarction, or ST elevation myocardial infarction involving the right ventricle/inferior wall (class IIa, level of evidence $\mathrm{C}$ ).

18. In AIS combined with recent myocardial infarction ( $>3$ months), if ST elevated, and involving the left ventricle/anterior wall, the safety and risk of IV rt-PA thrombolysis is uncertain (class IIb, level of evidence C).

19. In severe AIS with acute pericarditis which may lead to severe disability (modified Rankin Scale (mRS) score 3-5 points), the benefit of IV rt-PA thrombolysis is not clear. An urgent cardiologist consultation is required (class IIb, level of evidence C).

20. In mild or moderate AIS with acute pericarditis or left atrial/ventricular thrombus, the risk and benefit of IV rt-PA thrombolysis is unknown (class III, level of evidence $\mathrm{C}$ ).

21. Severe AIS with left atrial/ventricular thrombus, or atrial myxoma, or papillary fibroids may have severe disability (mRS score 3-5 points). The safety and efficacy of IV rt-PA are unknown (class IIb, level of evidence $\mathrm{C}$ ).

22. In AIS with cardiovascular or cerebrovascular digital subtraction angiography, IV rt-PA thrombolysis may be benefit. Patients should be carefully assessed for indications, contraindications and relative contraindications (class IIa, level of evidence A).

23. The efficacy and safety of IV rt-PA thrombolysis in patients with AIS with malignancy is unknown. If the expected survival period is $>6$ months, no other contraindications, no coagulation abnormalities or bleeding, careful consideration of IV rt-PA thrombolysis is suitable (class IIb, level of evidence $\mathrm{C}$ ).

24. Pregnant women with moderate or severe stroke may benefit from IV rt-PA thrombolysis if the benefits of IV thrombolysis outweigh the risk of uterine bleeding (class IIa, level of evidence $\mathrm{C}$ ).

25. The evidence of benefit and risk of IV rt-PA thrombolysis for patients with AIS within 14 days postpartum is insufficient (class IIb, level of evidence C).

\section{Patients within 6 hours after AIS—bridging/endovascular treatment.}

\section{Recommendations}

1. Mechanical thrombectomy is strongly recommended for patients within 6 hours after AIS if they meet all the following criteria: (1) prestroke mRS score of 0-1; (2) causative occlusion of the internal carotid artery (ICA) or middle cerebral artery (MCA) segment 1 (M1); (3) age $\geq 18$ years; (4) NIHSS score of $\geq 6$ and (5) ASPECTS of $\geq 6$ (class I, level of evidence A).

2. It is reasonable to initial treatment with intra-arterial thrombolysis within 6 hours after AIS caused by occlu- 
sions of the MCA for carefully selected patients who have contraindications or no clinical response to the use of IV rt-PA and could not perform mechanical thrombectomy (class IIa, level of evidence B).

3. Endovascular treatment should be performed as soon as possible after its indication. Patients eligible for IV rt-PA should receive IV rt-PA and direct perform bridging treatment for mechanical thrombectomy (class I, level of evidence A).

4. Mechanical thrombectomy should performed as the first-line treatment for patients who have contraindications to the use of IV rt-PA (class IIa, level of evidence A).

5. Stent retrievers is indicated for mechanical thrombectomy as first choice (class I, level of evidence A). Other thrombectomy or aspiration devices approved by local health authorities may be used at the operators' discretion (class IIa, level of evidence B).

6. Mechanical thrombectomy with stent retrievers may be reasonable for carefully selected patients with AIS in whom treatment can be initiated (groin puncture) within 6 hours of symptom onset and who have causative occlusion of the MCA segment 2 (M2) or MCA segment 3 (M3) portion of the MCAs (class IIb, level of evidence B).

7. Mechanical thrombectomy with stent retrievers may be reasonable for carefully selected patients with AIS in whom treatment can be initiated (groin puncture) within 6 hours of symptom onset and who have caus- ative occlusion of the anterior cerebral arteries, vertebral arteries, basilar artery or posterior cerebral arteries (class IIb, level of evidence C).

8. Mechanical thrombectomy with stent retrievers may be reasonable for patients with AIS in whom treatment can be initiated (groin puncture) within 6 hours of symptom onset and who have prestroke mRS score $>1$, ASPECTS $<6$ or NIHSS score $<6$, and causative occlusion of the ICA or proximal MCA (M1). Additional RCT data are needed (class IIb, level of evidence B).

\section{Patients within 6-24 hours after AIS—endovascular treatment.}

The treatment process after AIS is shown in figure 3 .

\section{Recommendations}

1. In selected patients with AIS within 6-16 hours of last known normal who have LVO in the anterior circulation and meet other DAWN or DEFUSE 3 eligibility criteria, mechanical thrombectomy is recommended (class I, level of evidence A).

2. In selected patients with AIS within 16-24hours of last known normal who have LVO in the anterior circulation and meet other DAWN eligibility criteria, mechanical thrombectomy is reasonable (class IIa, level of evidence B).

3. Patients with acute basilar artery occlusion within 6-24hours should be evaluated in centres with multimodal imaging and treated with mechanical throm-

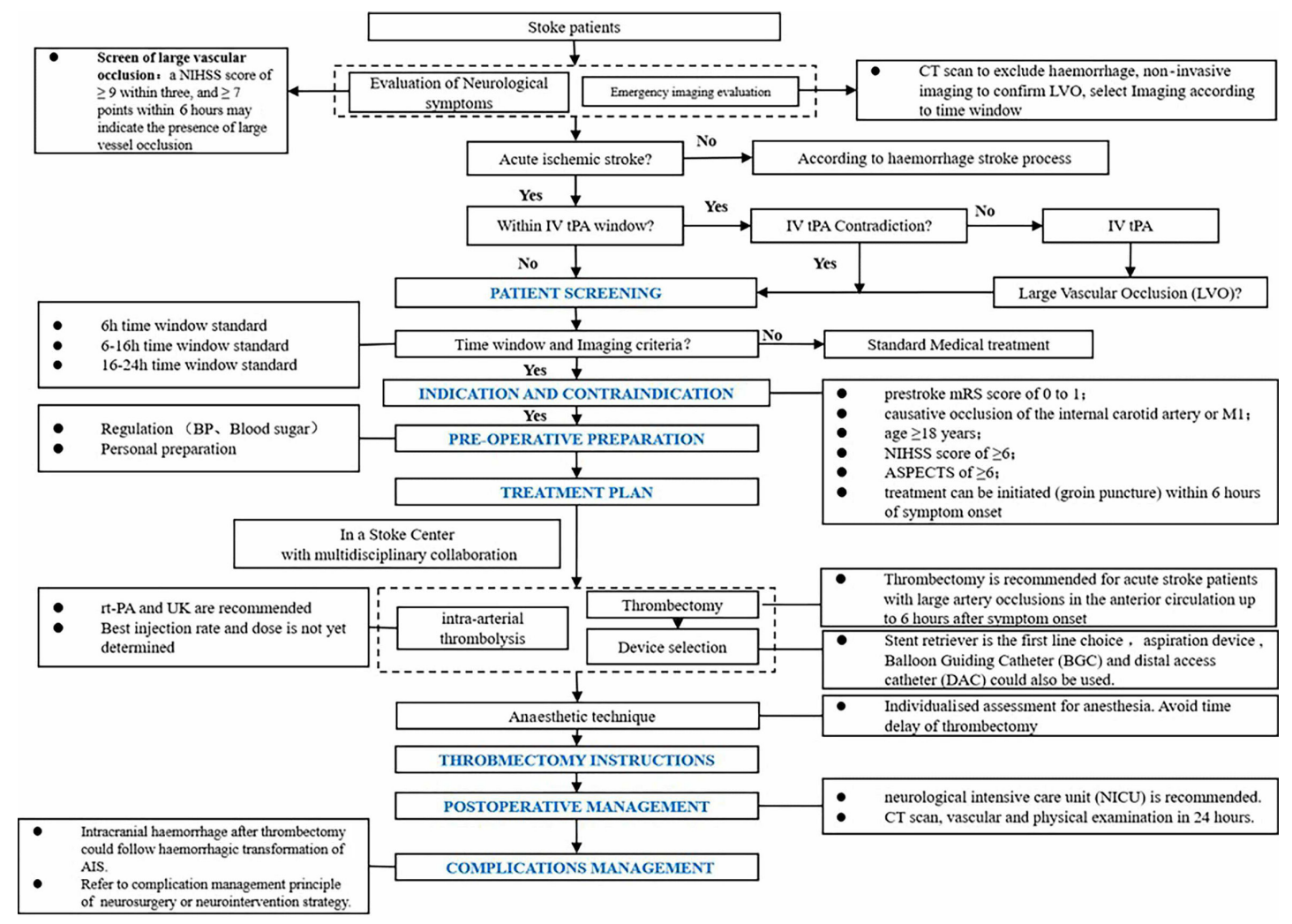

Figure 3 Endovascular treatment in patients with acute ischaemic stroke (AIS). BP, blood pressure; mRS, modified Rankin Scale; NIHSS, National Institutes of Health Stroke Scale; rt-PA, recombinant tissue plasminogen activator. 


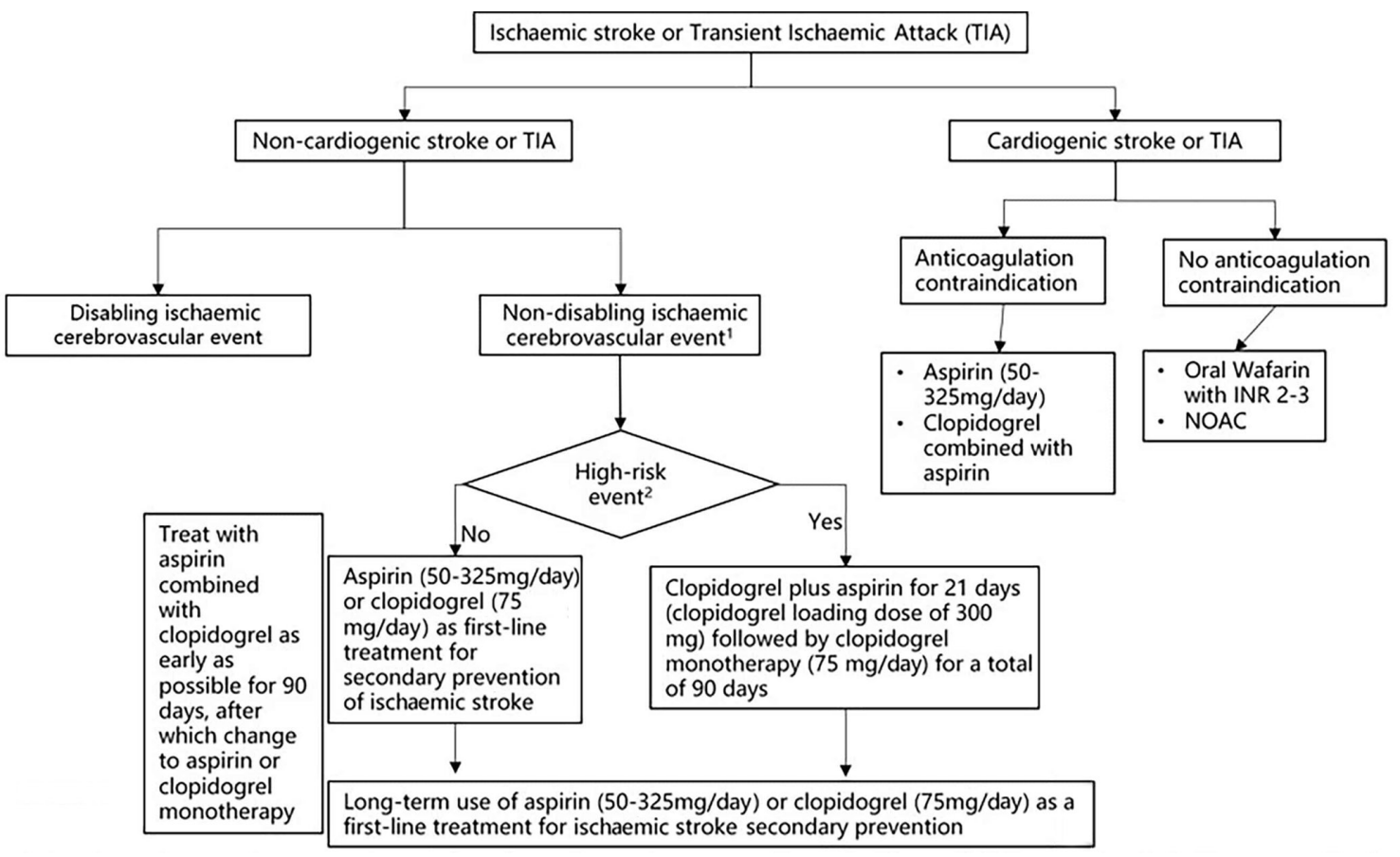

Figure 4 Antiplatelet treatment in patients with acute ischaemic stroke (AIS). INR, international normalised ratio; NOAC, novel oral anticoagulant. ${ }^{1}$ Non-disabling ischaemic cerebrovascular events refer to ischaemic cerebrovascular events without significant disability sequelae, including: 1. Transient ischemic attack (TIA) 2. Mild ischaemic stroke 3.Symptoms quickly relieved without significant disability sequelae. ${ }^{2}$ High-risk events include: 1 . High-risk TIA (ABCD $2 \geq 4$ points) and mild stroke with $24 \mathrm{~h}$ within onset. 2. Acute multiple cerebral infarction 3. Intracranial or extracranial aortic atherosclerotic stenosis $\geq 50 \%$.

bectomy or they may be treated within an RCT for thrombectomy approved by the local ethical committee (class IIb, level of evidence B).

4. The benefits of mechanical thrombectomy are uncertain for patients with acute large vascular occlusion for $>24$ hours (class IIb, level of evidence C).

\section{SECTION 4: ANTIPLATELET AGGREGATION THERAPY FOR ACUTE ISCHAEMIC CEREBROVASCULAR DISEASE}

By inhibiting one or more sites in the process of platelet aggregation, antiplatelet drugs can prevent thrombosis. At present, there are many RCTs on antiplatelet drugs in the world. Different kinds, doses and duration of antithrombotic regimens were used for specific groups of patients to obtain the best-individualised treatment. The latest research on single, double and triple antiplatelet drugs is introduced as below.

The antiplatelet aggregation treatment process for patients with AIS is shown in figure 4 .

\section{Single drug antiplatelet aggregation therapy}

Recommendations

1. Aspirin is recommended for patients with AIS within 24-48 hours after onset. For patients treated with IV rt$\mathrm{PA}$, aspirin is usually delayed until 24 hours later (class I, level of evidence A).

2. Aspirin (50-325 mg/day) or clopidogrel $(75 \mathrm{mg} /$ day) alone can be used as primary antiplatelet drug therapy (class I, level of evidence A).
3. Aspirin therapy is not recommended as an alternative therapy for patients with AIS who are suitable for IV rt-PA thrombolysis or mechanical thrombectomy (class III, level of evidence B).

4. Ticagrelor (instead of aspirin) is not recommended for acute mild stroke (class III, level of evidence B).

5. Cilostazol can be used in patients with AIS as an alternative to aspirin if aspirin or clopidogrel is not available (class IIa, level of evidence A).

6 . For high risk of aspirin intolerance (gastrointestinal adverse reactions or allergies, etc) in patients with ischaemic stroke, indobufen (100 mg per time, twice a day) is feasible (class IIb, level of evidence B).

7. Abciximab is not recommended for AIS (class III, level of evidence B).

8. Tirofiban is safe in the perioperative period for bridging therapy or endovascular therapy. The recommended dose is $0.1-0.2 \mu \mathrm{g} /(\mathrm{kg} \bullet \mathrm{min})$ and continuous infusion should be limited in 24 hours (class IIa, level of evidence B).

9. The efficacy of tirofiban and eptifibatide has not been fully determined, and further studies are needed to confirm (class IIb, level of evidence B).

\section{Dual antiplatelet aggregation therapy}

Recommendations

1. For patients with mild stroke and high-risk TIA who did not receive IV thrombolysis, dual antiplatelet therapy (aspirin $100 \mathrm{mg}$ /day, clopidogrel $75 \mathrm{mg}$ /day (first 
day load dose $300 \mathrm{mg}$ )) was initiated within 24 hours of onset and lasted for 21 days, then clopidogrel $75 \mathrm{mg}$ / day which could significantly reduce stroke recurrence for 90 days (class I, level of evidence A).

2. The efficacy of dipyridamole alone or dipyridamole combined with aspirin for preventing the recurrence of ischaemic stroke still needs RCTs to confirm (class IIb, level of evidence B).

\section{Triple antiplatelet aggregation therapy \\ Recommendations}

1. Triple antiplatelet aggregation therapy (aspirin, clopidogrel and dipyridamole) are not recommended for the treatment of acute non-cardiogenic stroke and TIA (class III, level of evidence B).

\section{SECTION 5: OTHER TREATMENT IN THE ACUTE PHASE}

Some scientists proposed that in addition to antiplatelet treatment, hypothermia, hyperbaric oxygen, hypertension, albumin and other treatments may also help to improve the prognosis of patients with ischaemic stroke in the acute phase.

\section{Recommendations}

1. Evidence in basic and preclinical studies suggests that neurovascular protection therapy may be beneficial, but has not been proved in clinical studies to improve prognosis of patients with AIS and reduce recurrence. More clinical research is still needed, some related researches are also in progress currently (class IIb, level of evidence B).

2. The efficacy of induced hypothermia in patients with ischaemic stroke is unclear and further studies are needed. Most studies have found that induced hypothermia was a risk for infection, including pneumonia. Induced hypothermia should be administered only in clinical trials (class IIb, level of evidence B).

3. Hyperbaric oxygen therapy is not recommended for patients with AIS unless it is caused by air embolism. Hyperbaric oxygen therapy is related to claustrophobia, middle ear barotrauma and the increased risk of seizures (class III, level of evidence B).

4. Mechanical augmentation of blood flow to treat patients with AIS has not been perfected, curative effect is not sure and only can be used in clinical trials (class IIb, level of evidence B).

5. Effectiveness of induced hypertension treatment in patients with AIS is not clear, it can only be used in the study of clinical research (class IIb, level of evidence B).

6. It is not recommended for routine use of blood volume expansion or haemodilution therapy in patients with AIS (class III, level of evidence A).

7. At present, there is no evidence that transcranial nearinfrared laser therapy for ischaemic stroke is beneficial, therefore using transcranial near-infrared laser treatment in ischaemic stroke is not recommended (class IIb, level of evidence B).
8. The routine use of high-dose albumin therapy in patients with AIS is not recommended (class III, level of evidence A).

\section{SECTION 6: GENERAL SUPPORTIVE CARE AND COMPLICATIONS MANAGEMENT}

Complications are common in patients with AIS, such as brain oedema, cerebral haemorrhage, epilepsy and so on. This section discusses the recommendations of general supportive care and management of neurological complications.

\section{General supportive care}

Airway support, ventilator assistance and supplemental oxygen

\section{Recommendations}

1. Airway support and ventilator assistance are recommended for the treatment of patients with acute stroke who have decreased consciousness or who have bulbar dysfunction that causes compromise of the airway (class I, level of evidence $\mathrm{C}$ ).

2. Supplemental oxygen should be provided to maintain oxygen saturation $>94 \%$ (class I, level of evidence $\mathrm{C}$ ).

3. Supplemental oxygen is not recommended in nonhypoxic patients with AIS (class III, level of evidence B).

\section{Temperature}

\section{Recommendations}

1. Sources of hyperthermia (temperature $>38^{\circ} \mathrm{C}$ ) should be identified and treated, and antipyretic medications should be administered to lower temperature in hyperthermic patients with stroke (class I, level of evidence C).

2. The benefit of induced hypothermia for treating patients with ischaemic stroke is not well established. Hypothermia should be offered only in the ongoing clinical trials (class IIb, level of evidence B).

\section{Nutrition}

\section{Recommendations}

1. Enteral diet should be started within 7 days of admission after an AIS (class I, level of evidence B).

2. For patients with dysphagia, it is reasonable to initially use nasogastric tube for feeding in the early phase of stroke (starting within the first 7 days) and to place percutaneous gastrostomy tubes in patients with longer anticipated persistent inability to swallow safely (>2-3 weeks) (class IIa, level of evidence $\mathrm{C}$ ).

3. Nutritional supplements are reasonable to consider for patients who are malnourished or at risk of malnourishment (class IIa, level of evidence B).

4. Implementing oral hygiene protocols to reduce the risk of pneumonia after stroke may be reasonable (class IIb, level of evidence B). 
Prediction of infections

\section{Recommendations}

1. Routine use of prophylactic antibiotics has not been shown to be beneficial (class III, level of evidence B).

2. Routine placement of indwelling bladder catheters should not be performed because of the associated risk of catheter-associated urinary tract infections (class III, level of evidence $\mathrm{C}$ ).

\section{Deep vein thrombosis prophylaxis}

\section{Recommendations}

1. In immobile patients with stroke without contraindications, intermittent pneumatic compression in addition to routine care (aspirin and hydration) is recommended over routine care to reduce the risk of deep vein thrombosis (class I, level of evidence B).

2. The benefit of prophylactic-dose subcutaneous heparin (unfractionated heparin (UFH or LMWH) in immobile patients with AIS is not well established (class IIb, level of evidence A).

3. When prophylactic anticoagulation is used, the benefit of prophylactic-dose LMWH over prophylactic-dose UFH is uncertain (class IIb, level of evidence B).

4. In ischaemic stroke, elastic compression stockings should not be used (class III, level of evidence B).

Rehabilitation

\section{Recommendations}

1. It is recommended that early rehabilitation for hospitalised patients with stroke be provided in environments with organised, multidisciplinary stroke care (class I, level of evidence A).
2. It is recommended that stroke survivors receive rehabilitation at an intensity commensurate with anticipated benefit and tolerance (class I, level of evidence B).

3. High-dose, very early mobilisation within 24 hours of stroke onset should not be performed because it can reduce the odds of a favourable outcome at 3 months (class III, level of evidence B).

4. It is recommended that all individuals with stroke be provided a formal assessment of their activities of daily living and instrumental activities of daily living, communication abilities and functional mobility before discharge from acute care hospitalisation and the findings be incorporated into the care transition and the discharge planning process (class I, level of evidence B).

5. A functional assessment by a clinician with expertise in rehabilitation is recommended for patients with an acute stroke with residual functional deficits (class I, level of evidence $\mathrm{C}$ ).

\section{Management of neurological complications \\ Brain swelling and occupation signs}

The treatment process of brain swelling/intracranial hypertension is shown in figure 5 .

\section{Recommendations}

1. Patients with major infarctions are at high risk of developing brain oedema and intracranial hypertension. Transfer of patients to intensive care unit should be considered. Measures to lessen the risk of oedema and close monitoring of patients for signs of neurological worsening during the first days after stroke are recommended (class I, level of evidence $\mathrm{C}$ ).

2. In patients $\leq 60$ years of age with unilateral MCA infarctions who deteriorate neurologically within

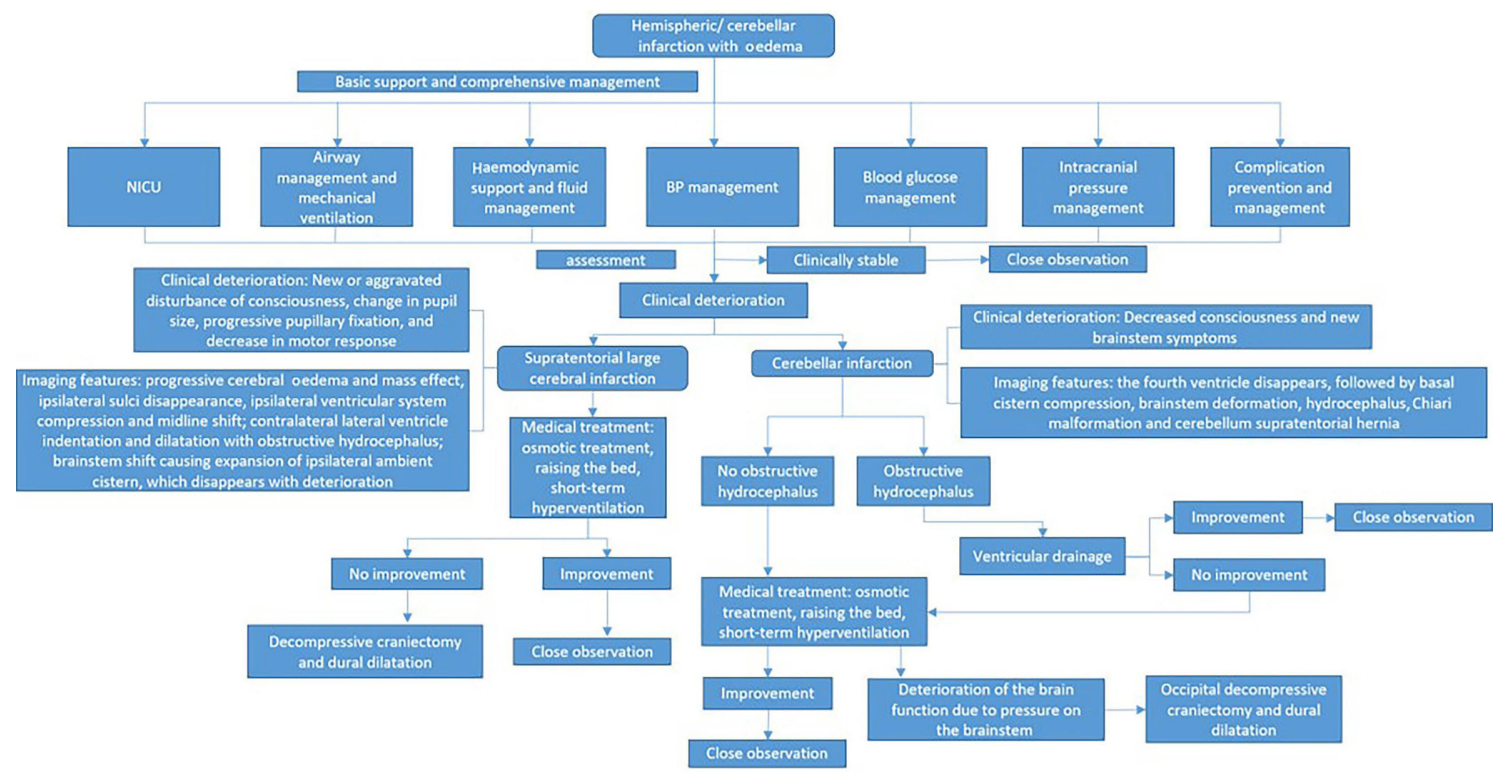

Figure 5 Management of cerebral oedema/high intracranial pressure in patients with acute ischaemic stroke. BP, blood pressure; NICU, neurological intensive care unit. 
48 hours despite medical therapy, decompressive craniectomy with dural expansion is reasonable (class IIa, level of evidence A).

3. In patients $>60$ years of age with unilateral MCA infarctions who deteriorate neurologically within 48 hours despite medical therapy, decompressive craniectomy with dural expansion may be considered (class IIb, level of evidence B).

4. Although the optimal trigger for decompressive craniectomy is unknown, it is reasonable to use a decrease in level of consciousness attributed to brain swelling as selection criteria (class IIa, level of evidence A).

5. Ventriculostomy is recommended in the treatment of obstructive hydrocephalus after a cerebellar infarct. Concomitant or subsequent decompressive craniectomy may or may not be necessary on the basis of factors such as infarct size, neurological condition, degree of brainstem compression and effectiveness of medical management (class I, level of evidence G).

6. Decompressive suboccipital craniectomy with dural expansion should be performed in patients with cerebellar infarction causing neurological deterioration from brainstem compression despite maximal medical therapy. When deemed safe and indicated, obstructive hydrocephalus should be treated concurrently with ventriculostomy (class I, level of evidence B).

7. Use of salvage osmotic therapy for patients with clinical deterioration from occupying signs associated with major supratentorial infarction or cerebellar infarction is reasonable (class IIa, level of evidence C).

8. Use of brief moderate hyperventilation $\left(\mathrm{PCO}_{2}\right.$ target $30-34 \mathrm{~mm} \mathrm{Hg}$ ) is a reasonable treatment for patients with acute severe neurological decline from brain swelling (class IIa, level of evidence C).

9. Hypothermia or barbiturates in the setting of ischaemic cerebral or cerebellar swelling is not recommended (class III, level of evidence B).

10. Because of a lack of evidence of efficacy and the potential to increase the risk of infectious complications, corticosteroids (in conventional or large doses) should not be administered for the treatment of cerebral oedema and increased intracranial pressure (class III, level of evidence A).

\section{Haemorrhagic transformation}

The treatment process of AIS haemorrhagic transformation is shown in figure 6 .

\section{Recommendations}

1. Symptomatic haemorrhagic transformation: stop using antithrombotic (antiplatelet, anticoagulation) (class I, level of evidence $\mathrm{C}$ ); for haemorrhage management associated with anticoagulation and thrombolysis, refer to cerebral haemorrhage treatment guidelines.

2. For patients with AIS with haemorrhagic transformation, starting or continuing antiplatelet or anticoagulant therapy should only be decided according to the specific clinical conditions and potential indications (class IIb, level of evidence B).

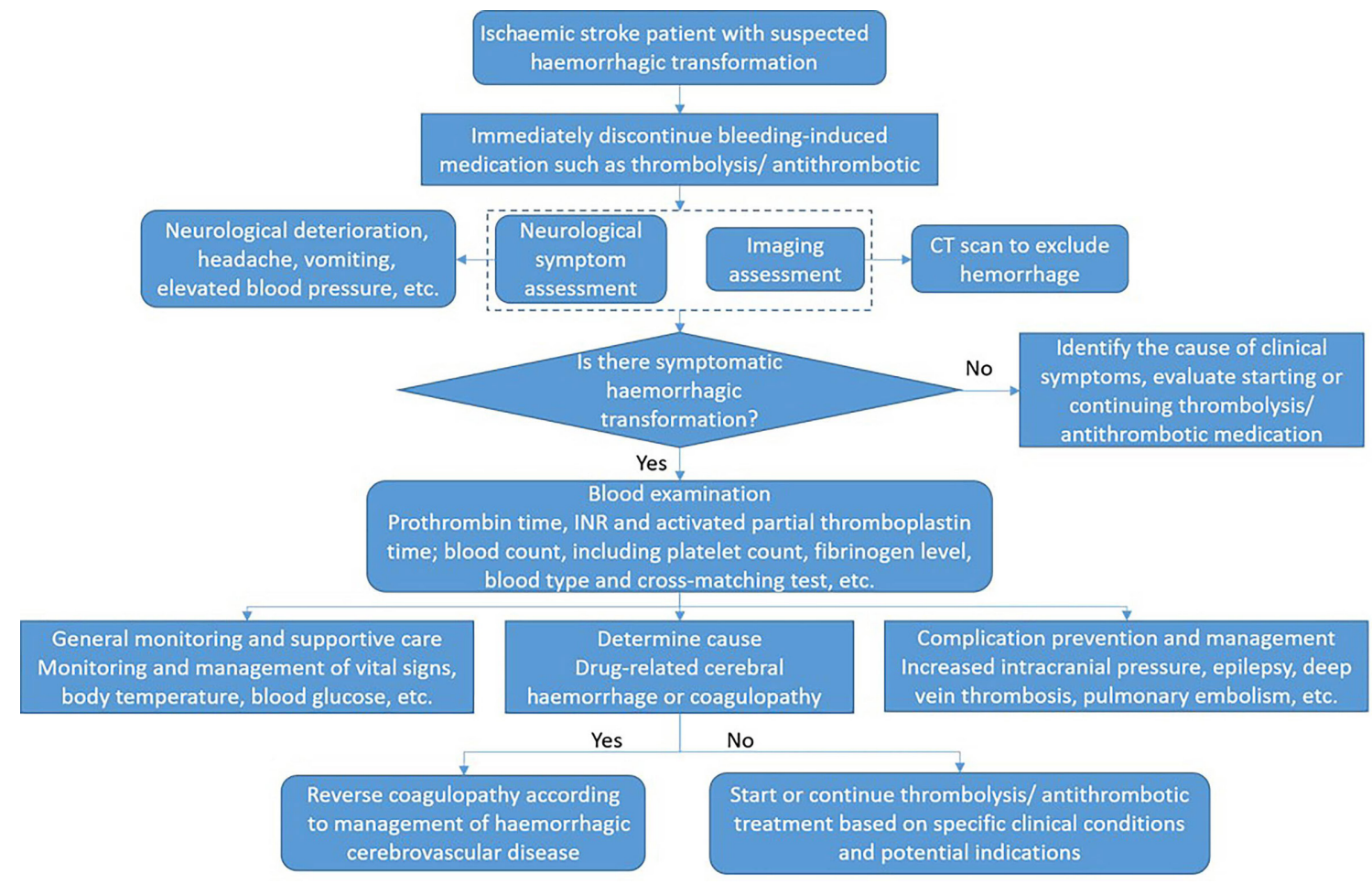

Figure 6 Haemorrhagic transformation management in patients with acute ischaemic stroke. INR, international normalised ratio. 


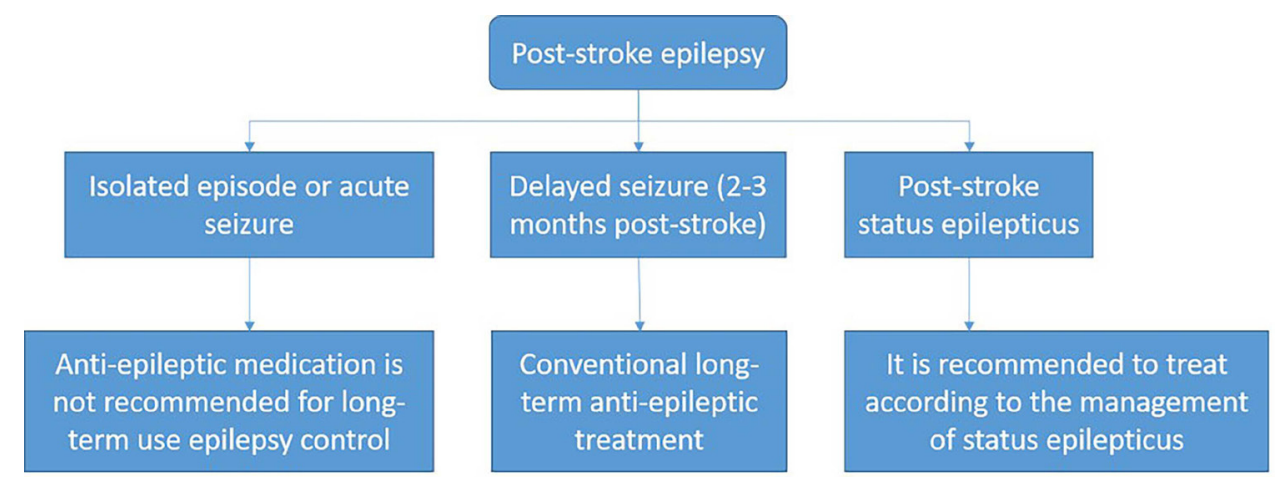

Figure 7 Epilepsy management within 24 hours after stroke onset.

\section{Seizures after AIS}

The treatment process of an initial seizure within 24 hours after AIS is shown in figure 7 .

\section{Recommendations}

1. Recurrent seizures after stroke should be treated in a manner similar to when they occur with other acute neurological conditions, and antiseizure drugs should be selected based on specific patient characteristics (class I, level of evidence C).

2. Prophylactic use of antiseizure drugs is not recommended (class III, level of evidence B).

\section{SECTION 7: EARLY EVALUATION AND DIAGNOSIS OF AETIOLOGY AND PATHOGENESIS OF ISCHAEMIC CEREBROVASCULAR DISEASE}

This section details the aetiology and pathogenesis diagnosis of ischaemic stroke from the aspects of examination methods and use of evaluation scales.

\section{Thorough inspection and evaluation are suggested}

Imaging examination of brain and blood vessels

\section{Recommendations}

1. All patients admitted to acute care hospital for ischaemic stroke should be evaluated by cranial imaging. For the majority of patients, the head CT scan is the first selection (class I, level of evidence B).

2. Early ischaemic signs or leukoaraiosis showed on CT cannot be an absolute exclusion standard for IV thrombolysis (class III, level of evidence B).

3. Routine use of MRI to identify intracranial microhaemorrhage, which can affect decisions to IV thrombolysis, is not recommended (class III, level of evidence B).

4. Patients with suspicious endovascular treatment should complete the non-invasive vascular evaluation as soon as possible, but should pay attention to avoid delaying thrombolysis (class I, level of evidence B).

5. Patients with suspected arterial occlusion and without previous renal impairment can conduct the head and neck CT angiography inspection directly, in order to avoid delay in treatment time in order to wait for creatinine to be resulted (class I, level of evidence B).
6. For patients who may need intravascular therapy, completing the evaluation of extracranial vessels including extracranial ICA and vertebral artery is helpful for guiding the treatment options (class IIa, level of evidence $\mathrm{C}$ ).

Cardiac examination (structure, rhythm, function and electrocardiogram activity)

Cardiac structure assessment

\section{Recommendations}

1. All patients with stroke should complete routine chest X-ray and transthoracic echocardiography in order to detect possible cardiac structural diseases (class I, level of evidence $\mathrm{C}$ ).

2. For patients with stroke with suspected cardiogenic embolism, performing transoesophageal ultrasonography to determine the presence of left auricular thrombosis, patent foramen ovale (PFO) or interatrial septal aneurysm is reasonable (class IIa, level of evidence B).

3. Transthoracic echocardiography cannot be replaced by transoesophageal echocardiography (class III, level of evidence $\mathrm{C}$ ).

4. Cardiac MRI is effective in identifying the aetiology of cryptogenic stroke. It can be carried out in hospitals which can do cardiac MRI (class IIb, level of evidence B).

5. Specific heart lesions found during cardiac screening in patients with stroke should be actively guided in specialised individual treatment (class I, level of evidence B).

\section{Cardiac rhythm assessment}

\section{Recommendations}

1. Asymptomatic atrial fibrillation and arrhythmia are very common, screening for atrial fibrillation should be routinely performed in the clinic, the routine checking of pulse should be performed on a patient $>65$ years of age and a 12-lead ECG should be conducted on patients with abnormalities of pulses (class I, level of evidence A).

2. The Congestive heart failure, Hypertension, Age $>75$, Diabetesmellitus, and prior Stroke or transient isch- 
emic attack (CHADS2) or Congestive Heart Failure, Hypertension, Age $\geq 75$ [Doubled], Diabetes Mellitus, Prior Stroke or Transient Ischemic Attack [Doubled], Vascular Disease, Age 65-74, Female (CHA2DS2-VASc) score is recommended for patients with persistent atrial fibrillation when assessing for the risk of stroke, and used to guide intervention (class I, level of evidence A).

3. In patients with latent stroke who may have embolism, 24 hours or long-term and remote cardiac monitoring aiming to find any paroxysmal atrial fibrillation is reasonable (class IIa, level of evidence B).

4. For patients with non-persistent atrial fibrillation or paroxysmal atrial fibrillation/atrial tachycardia (>5.5 hours) within 30 days or paroxysmal atrial fibrillation for $>30 \mathrm{~s}$, stroke prevention treatment in patient with persistent atrial fibrillation may be reasonable (class IIb, level of evidence B).

5. Whether arrhythmias other than atrial fibrillation or paroxysmal supraventricular tachycardia are associated with embolic events is unclear, and any intervention of those arrhythmias to reduce the incidence of embolism is still inadequate, symptomatic treatment can be taken into consideration (class III, level of evidence C).

\section{Cardiac function evaluation}

\section{Recommendations}

1. It is recommended to include cardiac function in the cardiac assessment of patients with stroke, especially the left atrium, left auricle and left ventricle function.

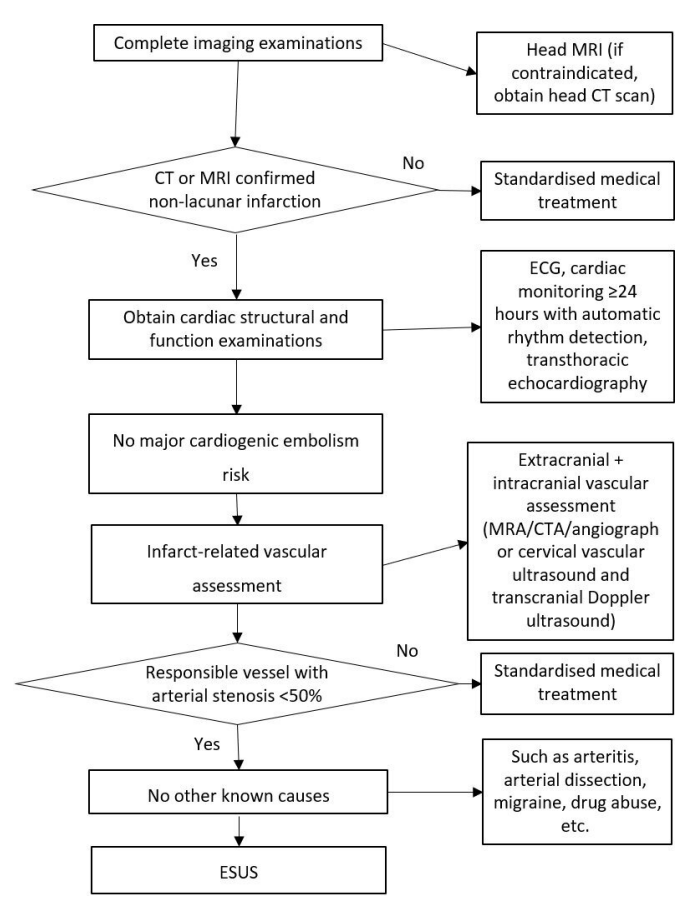

Figure 8 Diagnosis process for unexplained embolic stroke. CTA, CT angiography; ESUS, embolic stroke of undetermined source; MRA, magnetic resonance angiography.
Specific projects include volume index, emptying index and blood flow velocity (class I, level of evidence B).

2. The left atrium, left auricle and left ventricular blood flow disturbance, and left atrial spontaneous ultrasound contrast phenomenon are independent risk factors for embolus formation and triggering embolism. It is necessary to find the cause and take intervention measures actively (class IIa, level of evidence B).

Examination and evaluation of latent stroke and the diagnostic procedure for unexplained thrombotic stroke are shown in figure 8 .

\section{Risk factor assessment and risk stratification \\ Assessment of blood pressure}

\section{Recommendations}

1. Hypertension after AIS should be moderated strictly and lowered moderately, controlling the blood pressure in 140 160/80 99 mm Hg within 24 48 hours is reasonable (class I, level of evidence A).

2. Blood pressure variation and pulse pressure should be monitored closely after AIS. Blood pressure correlates the prognosis (class IIa, level of evidence B).

\section{Evaluation of blood lipid}

\section{Recommendations}

1. Dyslipidaemia (too high or too low) is closely related to poor prognosis. Serum lipid level should be actively assessed after AIS, in order to guide lipid-lowering treatment and secondary prevention (class IIa, level of evidence B).

2. Relatively low blood lipids may indicate that the condition of cerebral infarction is more serious, and attention should be paid to the changes of patients' condition (class IIb, level of evidence $\mathrm{C}$ ).

3. At present, the rate of reaching the standard of blood lipid control after AIS in China is still low, blood lipid control should be strengthened, at the same time pay attention to regular monitoring to avoid bleeding transformation (class I, level of evidence B).

\section{Assessment of abnormal glucose metabolism}

\section{Recommendations}

1. High blood sugar level and blood sugar fluctuation after AIS is closely related to the prognosis. Clinical monitoring and strict glycaemic control are recommended (class I, level of evidence A).

2. Blood glucose should be strictly monitored after AIS and insulin should be recommended for stable hyperglycaemic control, it is reasonable to control the blood glucose concentration to $5 \sim 8 \mathrm{mmol} / \mathrm{L}$ (class IIa, level of evidence B).

\section{Risk stratification assessment for TIA}

Risk stratification of TIA can use California risk score, the stroke prognosis instrument I (SPI- I) and SPI-II risk 
score, the Essen Stroke Risk Score (ESRS), the Age, Blood pressure, Clinical features, Duration of symptoms and Diabetes $\left(\mathrm{ABCD}_{2}\right)$ score to evaluate.

\section{Stratified assessment of ischaemic stroke}

Stratification of ischaemic stroke can be evaluated by atherosclerotic cardiovascular disease (ASCVD), ESRS, CHADS2 score and CHA2DS2-VASc score, the Hypertension, Abnormal Renal/Liver Function, Stroke, Bleeding History or Predisposition, Labile INR, Elderly, Drugs/ Alcohol Concomitantly (HAS-BLED) scale, SPI-II scale.

\section{SECTION 8: INTERVENTION ON AETIOLOGY AND PATHOGENESIS}

This chapter classifies ischaemic stroke by causes. It mainly enumerates atherosclerosis of large artery atherosclerosis, cardioembolic stroke, cerebral small vessel disease and other special causes. It is suggested that corresponding disease management and intervention measures should be taken for ischaemic stroke with different causes. Taking corresponding disease management and intervention measures for ischaemic stroke with different causes is helpful to improve the prognosis.

\section{Large artery atherosclerotic stroke \\ Antithrombotic therapy \\ Recommendations}

1. For patients with symptomatic intracranial artery stenosis, antiplatelet therapy should be started as soon as possible and used for long term. The alternative antiplatelet drugs are aspirin, clopidogrel and cilostazol (class I, level of evidence A).

2. For minor patients with stroke with high-risk intracranial artery stenosis (70\%-99\%), it is recommended to take single antiplatelet therapy after 90 days of dual antiplatelet therapy (aspirin and clopidogrel). And dual antiplatelet therapy combined with stent therapy in 90 days is not recommended (class III, level of evidence B).

3. For patients with stroke with intracranial arterial stenosis, aspirin plus clopidogrel is recommended to reduce the risk of early stroke recurrence caused by thromboembolism. One week later, the risk is reassessed for the purpose of determining whether to continue the combined treatment. The duration of dual antiplatelet therapy can last for 3 months after the onset of the disease. Routine anticoagulant therapy is not recommended for secondary prevention (class I, level of evidence A).

Surgical intervention: combined with intracranial and extracranial macrovascular stenosis and haemodynamic mechanism.

\section{Recommendations}

1. When clinical indicators or brain imaging demonstrate that the core of small infarction and at-risk area (penumbra) are caused by insufficient blood flow due to severe stenosis or occlusion of the carotid artery, or acute neurological impairment after carotid endarterectomy (CEA) with suspected acute thrombosis at the site of operation, the effectiveness of emergency CEA has not been confirmed (class II, level of evidence B).

2. The effectiveness of emergency CEA has not been proven in patients with unstable neurological status (such as progressive stroke) (class II, level of evidence B).

\section{Management of cardioembolic stroke \\ Timing of initiation of anticoagulant therapy}

\section{Recommendations}

1. For patients with non-massive cerebral infarction and not from cardiogenic embolism and without other bleeding risks, anticoagulant therapy is recommended and to be initiated within 2 weeks (class IIa, level of evidence B).

2. For patients at high risk of bleeding, or large infarction area or poor blood pressure control, the timing of initiation of anticoagulation therapy should be extended beyond 2 weeks (class IIa, level of evidence B).

3 . The size and severity of stroke should be taken into account when consider anticoagulation. It is suggested that anticoagulation can be initiated 1 day after TIA, 3 days after non-disabling small infarction and 6 days after moderate size infarction. Patients with large area infarction should wait at least 2-3 weeks (class IIa, level of evidence B).

4. For most patients with AIS with atrial fibrillation, it is reasonable to start oral anticoagulant therapy within 4-14 days after onset (class IIa, level of evidence B).

Drug selection

\section{Recommendations}

1. For patients with ischemic stroke or TIA with atrial fibrillation (including paroxysmal), appropriate doses of warfarin are recommended to prevent the recurrence of thromboembolism. The target dose of warfarin is to maintain INR at 2.0-3.0 (class I, level of evidence A).

2. New oral anticoagulants can be used as an alternative to warfarin. New oral anticoagulants include dabigatran, rivaroxaban, apixaban and edoxaban (class I, level of evidence A). Individual factors should be taken into account in the selection of drugs.

3. Emergency anticoagulant therapy for patients with AIS is not recommended for the prevention of early recurrence of stroke, stop the deterioration of neurological function and improve the outcome of AIS (class III, level of evidence A).

4. The effectiveness of emergency anticoagulant therapy is unclear in patients with AIS with severe ipsilateral ICA stenosis (class IIb, level of evidence A).

5. For patients with AIS with extracranial intravascular non-occlusive thrombosis, the safety and efficacy of short-term anticoagulant therapy are unclear (class IIb, level of evidence C). 
Etiological management

Atrial fibrillation

\section{Recommendations}

1. For patients with ischaemic stroke or TIA with atrial fibrillation, the time of anticoagulation should be chosen according to the severity of ischaemia and the risk of bleeding transformation. It is suggested that anticoagulation therapy should be given within 14 days after the onset of neurological symptoms to prevent stroke recurrence. For patients with high risk of bleeding, the timing of starting anticoagulation should be appropriately prolonged (class IIa, level of evidence B).

2. If patients with ischaemic stroke or TIA with atrial fibrillation are unable to receive oral anticoagulant therapy, aspirin alone may be considered for treatment (class IIa, level of evidence B). Aspirin combined with clopidogrel should be carefully selected as the choice of antiplatelet therapy (class IIb, level of evidence B).

\section{Other cardiogenic embolism}

\section{Recommendations}

1. In patients with no left ventricular mural thrombus, but with akinesis or dyskinesis of the anterior wall, oral anticoagulation therapy of warfarin for 3 months should be considered (target INR value $=2.5$, range 2.0-3.0) (class IIa, level of evidence B).

2. If there is no left ventricular mural thrombus, but no movement or abnormal movement of the anterior wall is found, oral anticoagulation therapy of warfarin for 3 months should also be considered (target INR value $=2.5$, range 2.0-3.0) (class IIa, level of evidence B).

3. For patients with ischaemic stroke or TIA with rheumatic mitral valve disease but without atrial fibrillation and other risk factors, such as carotid stenosis, oral anticoagulation therapy with warfarin is recommended (target INR=2.5, range 2.0-3.0) (class IIa, level of evidence B).

4. Patients with rheumatic mitral valve disease who have been treated with warfarin, routinely combining with antiplatelet therapy after ischaemic stroke or TIA is not recommended (class III, level of evidence C). However, aspirin antiplatelet therapy can be added when ischaemic stroke or TIA still occurs during the treatment of sufficient amount of warfarin (class IIa, level of evidence B).

5. Patients with ischaemic stroke or TIA with nonrheumatic mitral valve disease or other valve diseases (local aortic arch, mitral annulus calcification, mitral valve prolapse, etc) without atrial fibrillation may consider antiplatelet therapy (class IIa, level of evidence B).

6. For patients with ischaemic stroke or TIA and mechanical artificial heart valve, long-term warfarin oral anticoagulation therapy is recommended (INR 2/5-3.5) (class IIa, level of evidence B).
7. For patients with previous history of ischaemic stroke or TIA and mechanical artificial heart valves, if the risk of bleeding is low, aspirin can be used in addition to warfarin anticoagulation (class IIa, level of evidence B).

8. It is suggested that any decision on PFO closure should be made jointly by neurologists and cardiologists (class I, level of evidence A).

9. Before PFO closure, other known causes of ischaemic stroke (including monitoring arrhythmias) should be carefully excluded. The possibility of PFO correlation with the stroke, risk factors and lifestyle changes should be assessed. And communication between patients and multidisciplinary clinical teams should be involved in making the decision. For ischaemic stroke caused by $\mathrm{PFO}, \mathrm{PFO}$ closure can be performed to reduce the risk of stroke recurrence (class I, level of evidence A).

\section{Cerebral small vessel disease}

Recommendations

1. The mechanism of ischaemic stroke caused by small vascular disease is complex. At present, it is recommended to manage blood pressure, and use of aspirin, clopidogrel or cilostazol (class I, level of evidence B).

2. Cerebral small vessel disease leads to a significant decrease in the adaptability of brain tissue to the changes of excessive hypertension and hypotension. The blood pressure of patients should be closely monitored (class IIa, level of evidence B).

3. Control of systolic and diastolic pressure is the key factor to control the incidence and progression of cerebral small vessel disease (class IIa, level of evidence B).

4. It is necessary to monitor the 24 hours ambulatory blood pressure in patients with cerebral small vessel disease. When conditions permit, it is best to detect changes in blood pressure during head upright tilt test (class I, level of evidence B).

\section{Management of stroke with other special causes}

Recommendations

1. For patients with ischaemic stroke with defined aetiology, targeted aetiological treatment is needed (class I, level of evidence A).

2. For patients with AIS with extracranial carotid or vertebral artery dissection, antiplatelet or anticoagulant therapy for 3-6 months may be reasonable (class IIa, level of evidence B).

3. For patients with moyamoya disease, it is suggested that active drug treatment should be taken for underlying diseases or complications, and the risk factors of stroke should be effectively controlled and managed. It is necessary to choose the appropriate time and mode of operation according to the evaluation of patients (class IIa, level of evidence B).

4. The diagnosis of vascular inflammatory diseases in the central nervous system is difficult, and the aetiological treatment should be carried out on the basis of definite diagnosis (class IIa, level of evidence B). 


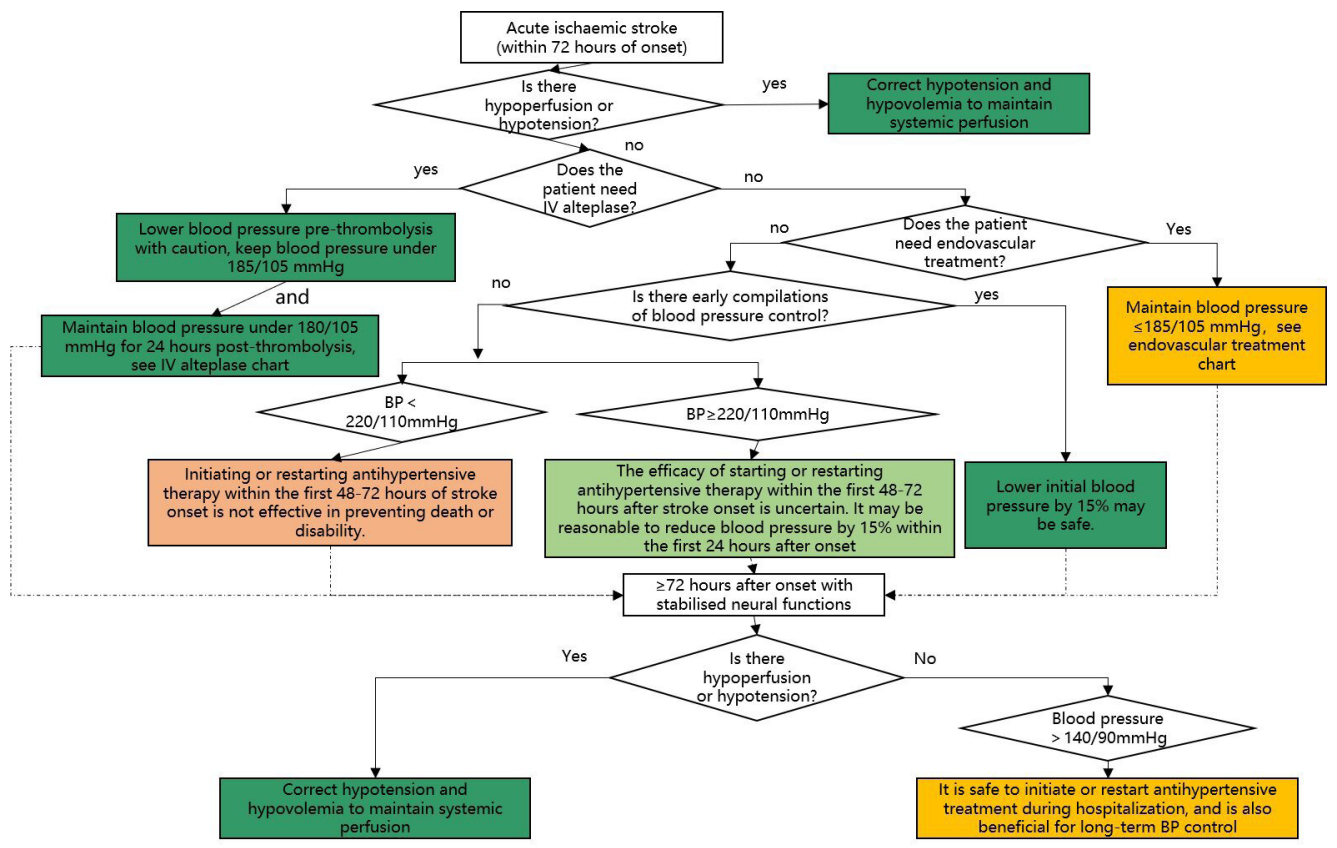

Figure 9 Blood pressure (BP) management in patients with acute ischaemic stroke (AIS).

5. For patients with suspected venous cerebral infarction, intracranial venous system angiography is recommended. Then, according to the clinical presentation and imaging results, these patients will receive the appropriate treatment (class IIa, level of evidence B).

\section{SECTION 9: MANAGEMENT OF RISK FACTORS AND LONG-TERM INTERVENTION}

The incidence of many patients with ischaemic cerebrovascular diseases is closely related to lifestyle and chronic diseases. Improving habits consciously and monitoring chronic diseases regularly helps patients' long-term survival.

\section{Blood pressure management}

The management of blood pressure within 72 hours after the onset of AIS is shown in figure 9 .

\section{Recommendations}

1. For patients with blood pressure $<220 / 120 \mathrm{~mm} \mathrm{Hg}$, who do not receive IV rt-PA or endovascular treatment and do not have complications requiring emergency antihypertensive treatment, starting or restarting antihypertensive therapy within the first 48-72 hours after AIS is not effective in preventing death or severe disability (class III, level of evidence A).

2. For patients with blood pressure $\geq 220 / 120 \mathrm{~mm} \mathrm{Hg}$, who do not receive IV rt-PA or endovascular treatment and do not have complications requiring emergency antihypertensive treatment, the effect of starting or restarting antihypertensive therapy within the first 4872 hours after AIS is uncertain. It may be reasonable to reduce blood pressure by $15 \%$ within the first 24 hours after a stroke attack (class IIb, level of evidence C).
3. For patients with AIS with other complications (such as simultaneous acute coronary events, acute heart failure, aortic dissection, bleeding transformation after thrombolysis or pre-eclampsia/eclampsia), early antihypertensive therapy is indicated. At the initial stage, a $15 \%$ reduction in blood pressure may be safe (class I, level of evidence $\mathrm{C}$ ).

4. Hypotension and hypovolaemia must be corrected after stroke to ensure systemic perfusion to support organ function (class I, level of evidence C).

5. For patients with AIS, the therapeutic effect of druginduced hypertension is uncertain (class IIb, level of evidence $\mathrm{C}$ ).

6. If the patient has stable neurological function during hospitalisation, but blood pressure $>140 / 90 \mathrm{~mm} \mathrm{Hg}$, it is safe to start or restart antihypertensive therapy. With the exception of contraindications, long-term control of blood pressure is reasonable (class IIa, level of evidence B).

\section{Management of abnormal lipid metabolism}

The comparison of the therapeutic dose of lipid-lowering drugs and the lipid-lowering management of AIS is shown in figure 10 .

\section{Recommendations}

1. Routine measurement of blood cholesterol levels is not recommended for all patients with atherosclerotic ischaemic stroke who are not on high-intensity statins (class III, level of evidence B).

2. For patients with ischaemic stroke during statins, it is reasonable to continue statins in the acute phase of stroke (class IIa, level of evidence B). 


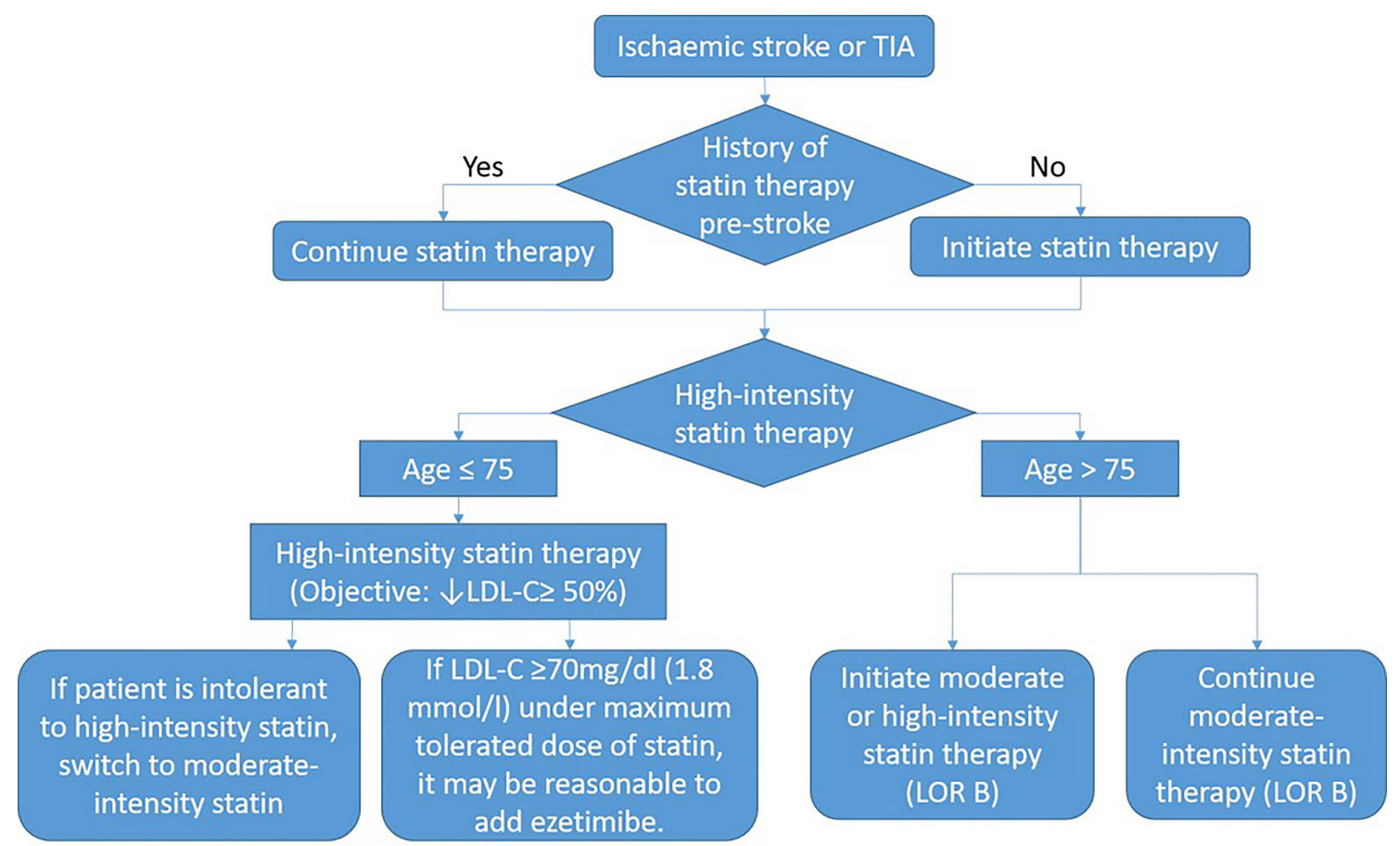

Figure 10 Blood pressure management in patients with acute ischaemic stroke. LDL-C, low-density lipoprotein-cholesterol; TIA, transient ischaemic attack; LOR B, Class IIB Recommendation .

3. For patients who meet the requirements for statins, it is reasonable to start statins during the hospital stay (class IIa, level of evidence $\mathrm{C}$ ).

4. It is suggested that LDL-C $<1.8 \mathrm{mmol} / \mathrm{L}(70 \mathrm{mg} / \mathrm{dL})$ should be used as a reference target for cholesterollowering therapy (class IIa, level of evidence C).

5. Atrial fibrillation is not a reason for not using statins in patients with ischaemic stroke (class IIa, level of evidence B).

6. High-intensity statins should be started or continued as a first-line treatment in females and $\leq 75$ years of age males with ASCVD, unless there are contraindications (class I, level of evidence A).

7. In patients with atherosclerotic ischaemic stroke who have already optimised statins, measuring blood cholesterol levels may help identify those who can benefit from proprotein convertase subtilisin/kexin type 9 (PCSK9) treatment (class IIb, level of evidence B).

8. For patients with poor lipid-lowering effect or intolerable of statins, lipid-lowering treatment can be combined with ezetimibe but regular monitoring of transaminase and physical examination should be done (class IIb, level of evidence B).

9. Patients with ASCVD with ischaemic stroke and other complications should be managed through lifestyle improvements, dietary advice and drug treatment (class I, level of evidence A).

10. For patients with ASCVD, it was originally intended to be treated with high-intensity statins. But when patients have contraindications or possible adverse reactions to statins, moderate-intensity statins should be used as a second option (class I, level of evidence A).

11. For patients over 75 years of age with clinical ASCVD, the benefits of reducing ASCVD risk, adverse drug reactions, drug-drug interactions and patient's wishes should be evaluated when initiating moderateintensity or high-intensity statins. It is reasonable to continue statins in patients who can tolerate (class IIb, level of evidence $\mathrm{C}$ ).

\section{Management of abnormal glucose metabolism}

\section{Recommendations}

1. The prognosis of persistent hyperglycaemia in patients with AIS within 24 hours after onset is worse than that of normal blood glucose. So, it is reasonable to control blood glucose to the range between $140 \sim 180 \mathrm{mg} /$ dL. At the same time, blood sugar should be closely monitored to prevent hypoglycaemia (class IIa, level of evidence $\mathrm{C}$ ).

2. Treatment should be given to patients with AIS with hypoglycaemia (blood glucose $<60 \mathrm{mg} / \mathrm{dL}$ ) (class I, level of evidence $\mathrm{C}$ ).

3. It is recommended that patients with ischaemic stroke or TIA with diabetes should be evaluated and given the best management guideline (class I, level of evidence A).

4. For all inpatients/outpatients with ischaemic stroke or TIA, rapid blood glucose, 2 hours postprandial blood glucose, glycosylated haemoglobin or $75 \mathrm{~g}$ oral glucose tolerance test are recommended for screening of diabetes mellitus (class IIa, level of evidence C). 
5. Lifestyle and/or drug intervention in patients with diabetes or prediabetes can reduce ischaemic stroke or TIA events. The recommended treatment target for glycosylated haemoglobin is $\leq 7 \%$ (class I, level of evidence B).

6 . The hypoglycaemic regimen should consider the clinical characteristics of patients and the safety of drugs. To set up individual blood glucose control targets, one should be on guard against the harm caused by hypoglycemia (class IIa, level of evidence B).

\section{Management of other risk factors \\ Recommendations}

1. Healthcare staff should strongly recommend that all patients with AIS who have smoked in the past year quit smoking (class I, level of evidence C).

2. Patients with AIS who smoke should consider starting intervention measures combined with drug therapy and behavioural support during hospitalisation (class IIb, level of evidence B).

3. Routine screening for obstructive sleep apnoea in patients with recent ischaemic stroke is not recommended (class III, level of evidence B).

4. For alcohol drinkers, it may be reasonable for men to drink $\leq 2$ units and non-pregnant women to drink $\leq 1$ unit per day (class IIb, level of evidence B).

5. The relationship between oral contraceptives and stroke needs to be further confirmed in prospective studies. Oral contraceptives may be associated with haemorrhagic stroke, which is more pronounced in patients with hypertension. So oral contraceptive is not recommended for patients with hypertension (class III, level of evidence C).

6. The relationship between drugs and stroke needs to be further studied. Drugs may be a risk factor for stroke and a factor for poor prognosis (class III, level of evidence $\mathrm{C}$ ).

7. For patients with recent ischaemic stroke or TIA and mild-to-moderate increase in blood homocysteine, folic acid, vitamin $B_{6}$ and vitamin $B_{12}$ supplementation can reduce the level of homocysteine. There is not enough evidence to support the practice of reducing homocysteine levels to reduce the risk of stroke recurrence (class IIb, level of evidence B).

\section{Author affiliations}

${ }^{1}$ Department of Neurology, Beijing Tiantan Hospital, Capital Medical University, Beijing, China

${ }^{2}$ Department of Neurology, Beijing Chaoyang Hospital, Capital Medical University, Beijing, China

${ }^{3}$ Department of Neurology, Peking Union Medical College Hospital, Beijing, China ${ }^{4}$ Department of Neurology, Guangzhou Overseas Chinese Hospital, The First Affiliated Hospital of JINAN University, Guangzhou, China

Collaborators Chairmen:Yongjun Wang, yongjunwang@ncrcnd.org.cn, Department of Neurology, Beijing Tiantan Hospital, Capital Medical University, Beijing, China; Jizong Zhao, zhaojz205@163.com / zhaojz@public.bta.net.cn, Department of Neurosurgery, Beijing Tiantan Hospital, Capital Medical University, Beijing, China. Vice-Chairmen: Qiang Dong, dong_qiang@fudan.edu.cn, Department of Neurology, Huashan Hospital, Fudan University, Shanghai, China; Anding Xu, tlii@jnu.edu.cn, Department of Neurology and Stroke Center, the First Affiliated
Hospital, Jinan University, Guangzhou, China. Members of Academic Committee: Kangning Chen, ckn_640827@126.com' Department of Neurology, The Southwest Hospital, the First Affiliated Hospital of Third Military Medical University, Chongqing, China; Junbo Ge, ge.junbo@zs-hospital.sh.cn' Shanghai Institute of Cardiovascular Diseases, Department of Cardiology, Zhongshan Hospital, Fudan University, Shanghai, China; Li Guo, guoli6@163.com, Department of Neurology, The Second Hospital of Hebei Medical University, Shijiazhuang, China; Li He, heli2003new@ 126.com, Department of Neurology, West China Hospital, Sichuan University, Chengdu, China; Bo Hu, hubo@hust.edu.cn, Department of Neurology, Union Hospital, Tongji Medical College, Huazhong University of Science and Technology (HUST), Wuhan, China; Yong Huo, huoyong@263.net.cn' Department of Cardiology, Peking University First Hospital, Beijing, China; Linong Ji, jiln@bjmu.edu.cn, Department of Endocrinology and Metabolism, Peking University People's Hospital, Medicine at Peking University, Beijing, China; Xunming Ji, robertjixm@hotmail.com / jixunming@vip.163.com, Department of Neurosurgery, Xuanwu Hospital, Capital University of Medicine, Beijing, China; Tielin Li, tielin2013@126.com / tielin.li@tom. com, Zhujiang Hospital of Southern Medical University, Guangzhou, China; Liping Liu, lipingsister@gmail.com, Department of Neurology, Beijing Tiantan Hospital, Capital Medical University, Beijing, China; Benyan Luo, luobenyan@zju.edu.cn, Department of Neurology, $1^{\text {st }}$ Affiliated Hospital of Zhejiang University, Hangzhou, China; Zhongrong Miao, zhongrongm@163.com, Department of Interventional Neuroradiology, Beijing Tiantan Hospital, Capital Medical University, Beijing, China; Xiaoyuan Niu, niuxiaoyuan1958@163.com, Department of Neurology, First Hospital of Shanxi Medical University, Taiyuan, China; Bin Peng, pengbin3@ hotmail.com; Department of Neurology, Peking Union Medical College Hospital, Peking Union Medical College and Chinese Academy of Medical Sciences, Beijing, China; Dingfeng Su, dfsu@smmu.edu.cn, Department of Pharmacology, the Second Military Medical University (SMMU), Shanghai, China; Beisha Tang, bstang7398@ 163.com, Department of Neurology, Xiangya Hospital, Central South University, Changsha, China; Chen Wang, wangchen-tr2002@163.com, Beijing Tiantan Hospital, Capital Medical University, Beijing, China; Ning Wang, nwang900@yahoo. com, Department of Neurology and Institute of Neurology, First Affiliated Hospital of Fujian Medical University, Fuzhou, China; Shuo Wang, captain9858@vip.sina. com, Department of Neurosurgery, Beijing Tiantan Hospital, Capital Medical University, Beijing, China; Wei Wang, wwang@vip.126.com / wwang@tjh.tjmu.edu. cn, Tongji Hospital, Tongji Medical College, Huazhong University of Science and Technology, Wuhan, China; Xin Wang, wang.xin@zs-hospital.sh.cn, Department of Neurology, Zhongshan Hospital, Fudan University, Shanghai, China; Yilong Wang, yilong528@aliyun.com, Department of Neurology, Beijing Tiantan Hospital, Capital Medical University, Beijing, China; Shizheng Wu, wushizheng2005@hotmail.com, Qinghai Province People's Hospital, Xining, China; Peng Xie, xiepeng@cqmu. edu.cn, Chongqing Medical University (CQMU), Chongqing, China; Yuming Xu, 13903711125@126.com / xym13903711125@126.com, Department of Neurology, the First Affiliated Hospital of Zhengzhou University, Zhengzhou, China; Yun Xu, xuyun20042001@aliyun.com, Department of Neurology, Drum Tower Hospital, Medical School of Nanjing University, Nanjing, China; Yi Yang, doctoryangyi@163. com / doctor_yangyi@hotmail.com, Department of Neurology, the First Hospital of Jilin University, Changchun, China; Jinsheng Zeng, zengjs@pub.guangzhou.gd. cn, Department of Neurology and Stroke Center, the First Affiliated Hospital of Sun Yat-Sen University, Guangdong, China; Chaodong Zhang, scdzhang@163.com, The First affiliated Hospital of China Medical University, Shenyang, China; Tong Zhang, zt61611@sohu.com, Capital Medical University School of Rehabilitation Medicine, China Rehabilitation Research Center, Beijing, China; Zhuo Zhang, zzhuo005@ gmail.com, Beijing Anzhen Hospital, Capital Medical University, Beijing, China; Gang Zhao, zhaogang@fmmu.edu.cn, Department of Neurology, Xijing Hospital, The 4th Military Medical University, Xi'an, China; Xingquan Zhao, zxq@vip.163. com, Department of Neurology, Beijing Tiantan Hospital, Capital Medical University, Beijing, China

Contributors $\mathrm{YW}^{1}$ and $\mathrm{LL}$ designed the protocol and framework, also participate in revision. WC and $\mathrm{HZ}^{2}$ drafted the sections of emergency assessment and diagnosis strategy and revised the whole manuscript. $\mathrm{XH}$ and $\mathrm{HZ}^{3}$ drafted the section of reperfusion therapy. WC and $\mathrm{HZ}^{2}$ drafted the section of antiplatelet aggregation therapy. WD drafted the section of other treatment in the acute phase and general supportive care and complications management. SL, WC and HL drafted the section of early evaluation and diagnosis of aetiology and pathogenesis. YL drafted the section of intervention on aetiology and pathogenesis. JX and HL drafted the section of management of risk factors and long-term intervention. $Y W^{4}, W X$ and $L H$ reviewed all the studies' design and interpretation, confirmed the level of evidence and classification. ${ }^{1}$ Yongjun Wang ${ }^{2}$ Hongyu Zhou ${ }^{3}$ Huaguang Zheng ${ }^{4}$ Yufei Wei

Funding This research received specific funding from Chinese Stroke Association Guidelines Writing Committee. 
Competing interests None declared.

Patient consent for publication Not required.

Provenance and peer review Not commissioned; externally peer reviewed.

Open access This is an open access article distributed in accordance with the Creative Commons Attribution Non Commercial (CC BY-NC 4.0) license, which permits others to distribute, remix, adapt, build upon this work non-commercially, and license their derivative works on different terms, provided the original work is properly cited, appropriate credit is given, any changes made indicated, and the use is non-commercial. See: http://creativecommons.org/licenses/by-nc/4.0/.

\section{ORCID iDs}

Liping Liu http://orcid.org/0000-0003-2943-055X

Weiqi Chen http://orcid.org/0000-0002-2551-9314

Hongyu Zhou http://orcid.org/0000-0002-9130-889X

Wanying Duan http://orcid.org/0000-0003-3527-9454

Xiaochuan Huo http://orcid.org/0000-0003-1264-5132

Huaguang Zheng http://orcid.org/0000-0001-6400-5474

Jingyi Liu http://orcid.org/0000-0001-7360-0267

Yufei Wei http://orcid.org/0000-0002-1822-7372

Jie Xu http://orcid.org/0000-0002-8320-218X

Yongjun Wang http://orcid.org/0000-0002-9976-2341

\section{REFERENCES}

1 Feigin VL, Mensah GA, Norrving B, et al. Atlas of the Global Burden of Stroke (1990-2013): The GBD 2013 study. Neuroepidemiology 2015;45:230-6.

2 Zhou M, Wang $\mathrm{H}$, Zhu J, et al. Cause-Specific mortality for 240 causes in China during 1990-2013: a systematic subnational analysis for the Global Burden of Disease Study 2013. Lancet 2016:387:251-72.

3 GBD 2016 Causes of Death Collaborators. Global, regional, and national age-sex specific mortality for 264 causes of death, 19802016: a systematic analysis for the Global Burden of Disease Study 2016. Lancet 2017;390:1151-210.

4 Wang W, Jiang B, Sun $\mathrm{H}$, et al. Prevalence, Incidence, and Mortality of Stroke in China: Results from a Nationwide Population-Based Survey of 480687 Adults. Circulation 2017;135:759-71.

5 Adams HP, Bendixen BH, Kappelle LJ, et al. Classification of subtype of acute ischemic stroke. Definitions for use in a multicenter clinical trial. TOAST. Trial of Org 10172 in Acute Stroke Treatment. Stroke 1993;24:35-41.

6 Arsava EM, Helenius J, Avery R, et al. Assessment of the Predictive Validity of Etiologic Stroke Classification. JAMA Neurol 2017;74:419-26.

7 The National Institute of Neurological Disorders and Stroke rt-PA Stroke Study Group. Tissue plasminogen activator for acute ischemic stroke. N Engl J Med 1995;333:1581-7.

8 Hacke W, Kaste M, Bluhmki E, et al. Thrombolysis with alteplase 3 to 4.5 hours after acute ischemic stroke. $N$ Engl J Med 2008;359:1317-29.

9 Sandercock P, Wardlaw JM, Lindley RI, et al. The benefits and harms of intravenous thrombolysis with recombinant tissue plasminogen activator within $6 \mathrm{~h}$ of acute ischaemic stroke (the third international stroke trial [IST-3]): a randomised controlled trial. Lancet 2012;379:2352-63.

10 Wardlaw JM, Murray V, Berge E, et al. Recombinant tissue plasminogen activator for acute ischaemic stroke: an updated systematic review and meta-analysis. Lancet 2012;379:2364-72.

11 Ogawa A, Mori E, Minematsu K, et al. Randomized trial of intraarterial infusion of urokinase within 6 hours of middle cerebral artery stroke: the middle cerebral artery embolism local fibrinolytic intervention trial (MELT) Japan. Stroke 2007;38:2633-9.

12 Saposnik G, Strbian D. Enlightenment and Challenges Offered by DAWN Trial (DWI or CTP Assessment With Clinical Mismatch in the Triage of Wake Up and Late Presenting Strokes Undergoing Neurointervention With Trevo). Stroke 2018;49:498-500.

13 Albers GW, Lansberg MG, Kemp S, et al. A multicenter randomized controlled trial of endovascular therapy following imaging evaluation for ischemic stroke (DEFUSE 3). Int J Stroke 2017;12:896-905.

14 Wang $Y$, Wang Y, Zhao X, et al. Clopidogrel with aspirin in acute minor stroke or transient ischemic attack. $N$ Engl J Med 2013;369:11-9.

15 Pan Y, Elm JJ, Li H, et al. Outcomes Associated With ClopidogrelAspirin Use in Minor Stroke or Transient Ischemic Attack: a Pooled Analysis of Clopidogrel in High-Risk Patients With Acute NonDisabling Cerebrovascular Events (CHANCE) and Platelet-Oriented Inhibition in New TIA and Minor Ischemic Stroke (POINT) Trials. JAMA Neurol 2019;76:1466-73.

16 Saxena M, Young P, Pilcher D, et al. Early temperature and mortality in critically ill patients with acute neurological diseases: trauma and stroke differ from infection. Intensive Care Med 2015;41:823-32.

17 den Hertog HM, van der Worp HB, van Gemert HM, et al. An early rise in body temperature is related to unfavorable outcome after stroke: data from the PAIS study. J Neurol 2011;258:302-7.

18 Roth EJ, Lovell L, Harvey RL, et al. Incidence of and risk factors for medical complications during stroke rehabilitation. Stroke $2001 ; 32: 523-9$

19 Dennis M, Sandercock P, Reid J, et al. Effectiveness of intermittent pneumatic compression in reduction of risk of deep vein thrombosis in patients who have had a stroke (CLOTS 3): a multicentre randomised controlled trial. Lancet 2013;382:516-24.

20 Hilker R, Poetter C, Findeisen N, et al. Nosocomial pneumonia after acute stroke: implications for neurological intensive care medicine. Stroke 2003;34:975-81.

$21 \mathrm{Xu} \mathrm{T}$, Zhang Y, Bu X, et al. Blood pressure reduction in acute ischemic stroke according to time to treatment: a subgroup analysis of the China Antihypertensive Trial in Acute Ischemic Stroke trial. J Hypertens 2017;35:1244-51.

22 Berge E, Cohen G, Lindley RI, et al. Effects of Blood Pressure and Blood Pressure-Lowering Treatment During the First 24 Hours Among Patients in the Third International Stroke Trial of Thrombolytic Treatment for Acute Ischemic Stroke. Stroke 2015;46:3362-9.

23 Yoshimura S, Uchida K, Daimon T, et al. Randomized Controlled Trial of Early Versus Delayed Statin Therapy in Patients With Acute Ischemic Stroke: ASSORT Trial (Administration of Statin on Acute Ischemic Stroke Patient). Stroke 2017;48:3057-63.

24 Larsson SC, Wallin A, Wolk A, et al. Differing association of alcohol consumption with different stroke types: a systematic review and meta-analysis. BMC Med 2016;14:178.

25 Cheng YC, Ryan KA, Qadwai SA, et al. Cocaine Use and Risk of Ischemic Stroke in Young Adults. Stroke 2016;47:918-22. 